\title{
The smallest strictly Neumaier graph and its generalisations
}

\author{
Rhys J. Evans* \\ School of Mathematical Sciences \\ Queen Mary University of London \\ Mile End Road \\ London E14NS, UK \\ r.evans@qmul.ac.uk \\ Sergey Goryainov ${ }^{\dagger}$ \\ School of Mathematical Sciences \\ Shanghai Jiao Tong University \\ 800 Dongchuan Road \\ Minhang District, Shanghai, China \\ Krasovskii Institute of Mathematics and Mechanics \\ S. Kovalevskaja st. 16 \\ Yekaterinburg, 620990, Russia \\ Faculty of Mathematics \\ Chelyabinsk State University \\ Brat'ev Kashirinyh st. 129 \\ Chelyabinsk, 454021, Russia \\ 44g@mail.ru

\section{Dmitry Panasenko} \\ Faculty of Mathematics \\ Chelyabinsk State University \\ Brat'ev Kashirinyh st. 129 \\ Chelyabinsk, 454021, Russia \\ makare95@mail.ru
}

Submitted: Sep 18, 2018; Accepted: Apr 11, 2019; Published: May 31, 2019

(c) The authors. Released under the CC BY-ND license (International 4.0).

\footnotetext{
${ }^{*}$ Corresponding author

†Supported by the NSFC (11850410430), STCSM (17690740800) and RFBR (17-51-560008).

${ }_{\ddagger}^{\ddagger}$ Supported by RFBR (17-51-560008).
} 


\begin{abstract}
A regular clique in a regular graph is a clique such that every vertex outside of the clique is adjacent to the same positive number of vertices inside the clique. We continue the study of regular cliques in edge-regular graphs initiated by A. Neumaier in the 1980s and attracting current interest. We thus define a Neumaier graph to be an non-complete edge-regular graph containing a regular clique, and a strictly Neumaier graph to be a non-strongly regular Neumaier graph. We first prove some general results on Neumaier graphs and their feasible parameter tuples. We then apply these results to determine the smallest strictly Neumaier graph, which has 16 vertices. Next we find the parameter tuples for all strictly Neumaier graphs having at most 24 vertices. Finally, we give two sequences of graphs, each with $i^{\text {th }}$ element a strictly Neumaier graph containing a $2^{i}$-regular clique (where $i$ is a positive integer) and having parameters of an affine polar graph as an edge-regular graph. This answers questions recently posed by G. Greaves and J. Koolen.
\end{abstract}

Mathematics Subject Classifications: 05C69, 05E30

\title{
1 Introduction
}

A regular clique, or more specifically an $m$-regular clique, in a regular graph $\Gamma$ is a clique $S$ such that every vertex of $\Gamma$ not in $S$ is adjacent to the same positive number $m$ of vertices of $S$. A regular clique can be equivalently viewed as a clique which is a part of an equitable 2-partition (see $[5,16]$ ), or a completely regular code of radius 1 (see [24] and [4, p. 345]). It is well known that a clique in a strongly regular graph is regular if and only if it is a Delsarte clique (see [1]; [4, Proposition 1.3.2(ii)]; [4, Proposition 4.4.6]).

In the early 1980s, A. Neumaier [23] studied regular cliques in edge-regular graphs, and a certain class of designs whose point graphs are strongly regular and contain regular cliques. He then posed the problem of whether there exists a non-complete, edge-regular, non-strongly regular graph containing a regular clique. We thus define a Neumaier graph to be a non-complete edge-regular graph containing a regular clique and define a strictly Neumaier graph to be a non-strongly regular Neumaier graph. (This is analoguous to the definitions of Deza graphs and strictly Deza graphs [12].)

Informed about the problem by L. Soicher in 2015, G. Greaves and J. Koolen then gave an answer by constructing an infinite family of strictly Neumaier graphs [19]. A. Gavrilyuk and S. Goryainov then searched for examples in a collection of known Cayley-Deza graphs [17], leading to the discovery of four more strictly Neumaier graphs. Recently, Greaves and Koolen [18] presented another infinite family of strictly Neumaier graphs, which contains one of the four strictly Neumaier graphs found by Gavrilyuk and Goryainov. Then Evans and Goryainov observed that the new infinite family of strictly Neumaier graphs can be generalised. Moreover, all four of the strictly Neumaier graphs found by Gavrilyuk and Goryainov are contained in the generalisation of the infinite family of graphs presented in $[18]$.

Further to a discussion with Koolen, Goryainov and his student D. Panasenko found the smallest strictly Neumaier graph, using methods similar to some of their work on Deza 
graphs. At roughly the same time, Evans found the smallest strictly Neumaier graph in a collection of vertex-transitive edge-regular graphs which had been provided by G. Royle [21]. Subsequent communications led to the collaboration found in the current paper.

In their paper [19], Greaves and Koolen pose two further questions about strictly Neumaier graphs which naturally arose from their work:

(A) [19, Question A] What is the minimum number of vertices for which there exists a non-strongly-regular, edge-regular graph having a regular clique?

(B) [19, Question B] Does there exist a non-strongly-regular, edge-regular graph having a regular clique with nexus greater than 2 ?

Indeed, before our work, all known strictly Neumaier graphs had at least 24 vertices, and contained $m$-regular cliques only for the value $m=1$.

In this paper we answer both of the above questions. In Section 3 we give some general results on Neumaier graphs and their feasible parameter tuples. In particular, we concentrate on conditions involving parameter tuples that force a Neumaier graph to be strongly regular. We also give a classification of Neumaier graphs with parameters achieving equality in a certain inequality. In Section 4 we apply the results found in Section 3 to determine the smallest strictly Neumaier graph, which turns out to be vertextransitive and has 16 vertices, valency 9 and a 2-regular 4-clique.

In Section 5 we present two new infinite sequences of strictly Neumaier graphs. Each of these sequences has first element the unique smallest strictly Neumaier graph. The $i^{\text {th }}$ element of each of these sequences is a strictly Neumaier graph which contains a $2^{i}$-regular clique. In fact, all of these graphs contain a subgraph isomorphic to a clique extension (see the definition of a clique extension in $[4$, p. 6]) of the unique smallest strictly Neumaier graph. These constructions show that the nexus of a clique in a strictly Neumaier graph is not bounded above by some constant number. Furthermore, each of the graphs in these sequences has the edge-regular graph parameters of an affine polar graph.

\section{Preliminaries}

In this paper we only consider finite, undirected graphs that contain no loops or multiple edges. Let $\Gamma$ be such a graph. We denote by $V(\Gamma)$ the vertex set of $\Gamma$, and $E(\Gamma)$ the edge set of $\Gamma$. For a vertex $u \in V(\Gamma)$, we define the neighbourhood of $u$ in $\Gamma$ to be the set $\Gamma(u)=\{w \in V(\Gamma): u w \in E(\Gamma)\}$. The complement of a graph $\Gamma$, denoted by $\bar{\Gamma}$, is the graph with vertex set $V(\bar{\Gamma})=V(\Gamma)$, and for distinct vertices $u, w \in V(\Gamma)$, we have $u w \in E(\bar{\Gamma})$ if and only of $u w \notin E(\Gamma)$.

Let $\Gamma$ be a graph and $v=|V(\Gamma)|$. The graph $\Gamma$ is said to be $k$-regular if every vertex has neighbourhood of size $k$. The graph $\Gamma$ is called regular if there exists a value $k$ such that $\Gamma$ is $k$-regular. The graph $\Gamma$ is edge-regular if it is non-empty, $k$-regular, and every pair of adjacent vertices have exactly $\lambda$ common neighbours. Then $\Gamma$ is said to be edge-regular with parameters $(v, k, \lambda)$, and refer to this as a parameter tuple. Denote by $E R G(v, k, \lambda)$ the set of edge-regular graphs with parameters $(v, k, \lambda)$. The graph $\Gamma$ is co-edge-regular if 
it is non-complete, $k$-regular and every pair of distinct non-adjacent vertices have exactly $\mu$ common neighbours. Then $\Gamma$ is said to be co-edge-regular with parameters $(v, k, \mu)$. The graph $\Gamma$ is strongly regular if it is both edge-regular and co-edge-regular. If $\Gamma$ is edge-regular with parameters $(v, k, \lambda)$, and co-edge-regular with parameters $(v, k, \mu)$, the graph is said to be strongly regular with parameters $(v, k, \lambda, \mu)$.

A clique in a graph $\Gamma$ is a set of pairwise adjacent vertices of $\Gamma$, and a clique of size $s$ is called an $s$-clique. A clique $S$ in a regular graph $\Gamma$ is regular if every vertex not in $S$ is adjacent to the same number $m>0$ of vertices in $S$. In this case we say that $S$ has nexus $m$ and is $m$-regular. Let us give several examples of strongly regular graphs containing a regular clique.

Example 1. Let $K_{r \times t}$ be the complete multipartite graph which has $r$ parts of size $t$. Let $S$ be a set consisting of exactly one vertex from each part of $\Gamma$. Then $S$ is a $(r-1)$-regular $r$-clique.

Example 2. For $n \geqslant 2$, the square lattice graph $L_{2}(n)$ has vertex set $\{1,2, \ldots, n\} \times$ $\{1,2, \ldots, n\}$, and two distinct vertices are joined by an edge precisely when they have the same value at one coordinate. This graph is strongly regular with parameters $\left(n^{2}, 2(n-\right.$ $1), n-2,2)$. Let $S$ be a set consisting of all vertices of $L_{2}(n)$ which have the same fixed value at the same fixed coordinate. Then $S$ is a 1 -regular $n$-clique.

Example 3. For $n \geqslant 3$, the triangular graph $T(n)$ has vertex set consisting of the subsets of $\{1,2, \ldots, n\}$ of size 2 , and two distinct vertices $A, B$ are joined by an edge precisely when $|A \cap B|=1$. This graph is strongly regular with parameters $\left(\left(\begin{array}{l}n \\ 2\end{array}\right), 2(n-2), n-2,4\right)$. Let $S$ be a set consisting of all vertices of $T(n)$ which contain a fixed element from $\{1,2, \ldots, n\}$. Then $S$ is a 2 -regular $(n-1)$-clique.

A Neumaier graph is a non-complete edge-regular graph which contains a regular clique. We denote by $N G(v, k, \lambda ; m, s)$ the set of Neumaier graphs which are edge-regular with parameters $(v, k, \lambda)$, and contain an $m$-regular $s$-clique, where $s \geqslant 2$. A strictly Neumaier graph is a Neumaier graph which is not strongly regular (the definition of a strictly Neumaier graph is analoguous to the definition of a strictly Deza graph, see [12]).

The tuple $(v, k, \lambda)$ is said to be extremal if $\operatorname{ERG}(v, k, \lambda)$ is non-empty and contains only strongly regular graphs. Similarly, the tuple $(v, k, \lambda ; m, s)$ is said to be extremal if $N G(v, k, \lambda ; m, s)$ is non-empty and contains only strongly regular graphs.

To answer Question A, we collect a series of conditions on the parameters $(v, k, \lambda ; m, s)$ that force at least one of the following to occur;

1. $\operatorname{ER} G(v, k, \lambda)$ is empty.

2. $(v, k, \lambda)$ is extremal.

3. $N G(v, k, \lambda ; m, s)$ is empty.

4. $(v, k, \lambda ; m, s)$ is extremal.

In the current paper, we will present both new and known results on extremal parameter tuples $(v, k, \lambda)$ and $(v, k, \lambda ; m, s)$. Conditions for a parameter tuple $(v, k, \lambda)$ to be extremal is an interesting area of study, and many instances of this type of extremality condition can be found in the literature (for example, in [7] or [4, Section 1.4]). 


\section{$2.1 \quad$ Edge-regular graphs}

First we state simple results concerned with taking the complement of the graphs we work with.

Lemma 2.1. The following statements hold.

(i) Suppose $\Gamma$ is a k-regular graph. Then $\bar{\Gamma}$ is a $(v-k-1)$-regular graph.

(ii) Suppose $\Gamma$ is an edge-regular graph with parameters $(v, k, \lambda)$. Then $\bar{\Gamma}$ is co-edgeregular, with parameters $(v, v-k-1, v-2 k+\lambda)$.

(iii) Suppose $\Gamma$ is a co-edge-regular graph with parameters $(v, k, \mu)$. Then $\bar{\Gamma}$ is edgeregular, with parameters $(v, v-k-1, v-2-2 k+\mu)$.

Corollary 2.2. Let $\Gamma$ be a strongly regular graph with parameters $(v, k, \lambda, \mu)$. Then $\bar{\Gamma}$ is strongly regular with parameters $(v, v-k-1, v-2-2 k+\mu, v-2 k+\lambda)$.

The next lemma gives basic properties of an edge-regular graph.

Lemma 2.3 ([4], Chapter 1$)$. Let $\Gamma$ be an edge-regular graph with parameters $(v, k, \lambda)$. Then:

(i) $v>k>\lambda$;

(ii) $v \geqslant 2 k-\lambda$;

(iii) 2 divides $v k$;

(iv) 2 divides $k \lambda$;

(v) 6 divides $v k \lambda$.

\section{$2.2 \quad$ Edge-regular graphs with regular cliques}

Let $\Gamma$ be an edge-regular graph with parameters $\tau=(v, k, \lambda)$. Our main tool in the investigation of Neumaier graphs is the clique adjacency polynomial, which is defined in [27], and given by

$$
C_{\tau}(x, y):=x(x+1)(v-y)-2 x y(k-y+1)+y(y-1)(\lambda-y+2) .
$$

The following theorem uses the clique adjacency polynomial to give a criterion for when any $s$-clique in any graph $\Gamma$ from $E R G(v, k, \lambda)$ is $m$-regular.

Lemma 2.4 ([28], Theorem 3.1). Let $\Gamma$ be a graph in $E R G(v, k, \lambda)$ having an s-clique $S$, with $s \geqslant 2$. If $m$ is a positive integer then

$$
C_{\tau}(m-1, s)=C_{\tau}(m, s)=0
$$

if and only if $S$ is an m-regular clique.

Further, we list several more tools which we use in the investigation of Neumaier graphs. The next result gives arithmetic conditions on the parameters of a Neumaier graph. By analysing these relations further, we reconstruct $s$ and $m$ as functions of $v, k, \lambda$. The property of these expressions to be integral numbers can then be seen as necessary conditions for an edge-regular graph to contain a regular clique. 
Lemma 2.5. Let $\Gamma$ be a graph in $N G(v, k, \lambda ; m, s)$. Then:

(i) $(v-s) m=(k-s+1) s$;

(ii) $(k-s+1)(m-1)=(\lambda-s+2)(s-1)$;

(iii) $s$ is the largest root of the polynomial

$$
(v-2 k+\lambda) y^{2}+\left(k^{2}+3 k-\lambda-v(\lambda+2)\right) y+v(\lambda+1-k)
$$

(iv) $m$ is the largest root of the polynomial

$$
(v-s) x^{2}-(v-s) x-s(s-1)(\lambda-s+2) .
$$

Proof.

(i) By Lemma 2.4, $C_{\tau}(m, s)=C_{\tau}(m-1, s)=0$ where $\tau=(v, k, \lambda)$. Then (i) is found by evaluating $0=C_{\tau}(m, s)-C_{\tau}(m-1, s)$.

(ii) Take the equation in (i) and substitute into $C_{\tau}(m, s)$.

(iii) Multiply the expression in (ii) by $(v-s)$ and use (i) to substitute for $(v-s) m$. We see that $s$ is a root of the polynomial. Note that $v \geqslant 2 k-\lambda$ and $v(\lambda+1-k) \leqslant 0$ by Lemma 2.3. This means there is at most one positive root to the polynomial.

(iv) Multiply the expression in (i) by $(m-1)$ and use (ii) to substitute for $(k-s+1)(m-1)$. We see that $m$ is a root of the polynomial. Note that $\lambda-s+2 \geqslant 0$ as an edge in an $s$-clique is in at least $s-2$ triangles of the graph $\Gamma$. This means there is at most one positive root of the polynomial.

Now we present a collection of results giving properties of all regular cliques in a Neumaier graph.

Lemma 2.6 ([23], Theorem 1.1). Let $\Gamma$ be a graph in $N G(v, k, \lambda ; m, s)$. Then:

(i) the maximum size of a clique in $\Gamma$ is $s$;

(ii) all regular cliques in $\Gamma$ are $m$-regular cliques;

(iii) the regular cliques in $\Gamma$ are precisely the cliques of size $s$.

We finish this section by giving a lower bound on the size of a regular clique in a strictly Neumaier graph. We can understand such a result as saying the following: Take a parameter tuple $(v, k, \lambda ; m, s)$ where $s$ is less than the bound. Then the parameters $(v, k, \lambda ; m, s)$ are extremal.

Lemma 2.7 ([19], Prop. 4.2). Let $\Gamma$ be a strictly Neumaier graph from $N G(v, k, \lambda ; m, s)$. Then $s \geqslant 4$, and consequently, $\lambda \geqslant 2$. 


\subsection{Affine polar graphs $\mathrm{VO}^{+}(2 e, 2)$}

Let $V$ be a $(2 e)$-dimensional vector space over a finite field $\mathbb{F}_{q}$, where $e \geqslant 2$ and $q$ is a prime power, provided with the hyperbolic quadratic form $Q(x)=x_{1} x_{2}+x_{3} x_{4}+\ldots+$ $x_{2 e-1} x_{2 e}$. The set $Q^{+}$of zeroes of $Q$ is called the hyperbolic quadric, where $e$ is the maximal dimension of a subspace in $Q^{+}$. A generator of $Q^{+}$is a subspace of maximal dimension $e$ in $Q^{+}$.

Lemma 2.8 ([3, Theorem 7.130]). Given an $(e-1)$-dimensional subspace $W$ of $Q^{+}$, there are precisely two generators that contain $W$.

Denote by $\mathrm{VO}^{+}(2 e, q)$ the graph on $V$ with two vectors $x, y$ being adjacent if and only if $Q(x-y)=0$. The graph $V^{+}(2 e, q)$ is known as an affine polar graph (see $[3,5,6]$ ).

Lemma 2.9. The graph $\mathrm{VO}^{+}(2 e, q)$ is a vertex-transitive strongly regular graph with parameters

$$
\begin{aligned}
v & =q^{2 e} \\
k & =\left(q^{e-1}+1\right)\left(q^{e}-1\right) \\
\lambda & =q\left(q^{e-2}+1\right)\left(q^{e-1}-1\right)+q-2 \\
\mu & =q^{e-1}\left(q^{e-1}+1\right) .
\end{aligned}
$$

Note that $\mathrm{VO}^{+}(2 e, q)$ is isomorphic to the graph defined on the set of all $(2 \times e)$ matrices over $\mathbb{F}_{q}$ of the form

$$
\left(\begin{array}{cccc}
x_{1} & x_{3} & \ldots & x_{2 e-1} \\
x_{2} & x_{4} & \ldots & x_{2 e}
\end{array}\right)
$$

where two matrices are adjacent if and only if the scalar product of the first and the second rows of their difference is equal to 0 .

Lemma 2.10. There is a one-to-one correspondence between cosets of generators of $Q^{+}$ and maximal cliques in $\mathrm{VO}^{+}(2 e, q)$.

Proof. Since $\mathrm{VO}^{+}(2 e, q)$ is vertex-transitive and the additive shift by an element is an automorphism of $\mathrm{VO}^{+}(2 e, q)$, it suffices to prove that every maximal clique containing the zero vector is a generator. The fact that every maximal clique containing the zero vector is a generator can be seen from [3, Theorem 7.3], [3, Corollary 7.16] and [3, Corollary $7.137]$.

Lemma 2.11. Every maximal clique in $\mathrm{VO}^{+}(2 e, q)$ is a $q^{e-1}$-regular $q^{e}$-clique.

Proof. This follows from Lemma 2.9, Lemma 2.10 and [4, Proposition 1.3.2(ii)].

A spread in $\mathrm{VO}^{+}(2 e, q)$ is a set of $q^{e}$ disjoint maximal cliques that correspond to all cosets of a generator. 


\section{Conditions on parameters to be extremal}

We will now give a collection of conditions on parameter tuples to show they are extremal. We first consider the tuples associated with edge-regular graphs, and then consider the tuples associated with Neumaier graphs.

\subsection{The triple of parameters $(v, k, \lambda)$}

When the triple $(v, k, \lambda)$ is extremal, there are no edge-regular graphs in $\operatorname{ER} G(v, k, \lambda)$ which are not strongly regular. Thus there is no strictly Neumaier graph with these edgeregular parameters. This fact will be heavily used when analysing the smallest Neumaier graph.

The following lemma gives a list of sufficient conditions for $(v, k, \lambda)$ to be extremal.

Lemma 3.1. Suppose $E R G(v, k, \lambda)$ is non-empty for some $v, k, \lambda$. Then the triple $(v, k, \lambda)$ is extremal if at least one of the following holds:

(i) $v=2 k-\lambda$.

(ii) $v=2 k-\lambda+1$.

(iii) There is a strongly regular graph with parameters $(v, v-k-1,0, v-2 k+\lambda)$.

Proof.

(i) These graphs are exactly the graphs $K_{s \times t}$ (see [28, Theorem 4.1]).

(ii) Take an edge-regular graph with parameters $(v, k, \lambda)$, with $v-2 k+\lambda=1$. By Lemma 2.1, we see that $\bar{\Gamma}$ is co-edge-regular with parameters $(v, v-k-1,1)$. Then by [4, Lemma 1.1.3], $\bar{\Gamma}$ is strongly regular. Thus $\Gamma$ is strongly regular.

(iii) Let $\Delta$ be a strongly regular graph with parameters $(v, v-k-1,0, v-2 k+\lambda)$. By Corollary 2.2, $\bar{\Delta}$ is strongly regular with parameters $(v, k, \lambda, \mu)$. A standard counting argument (see [4, Lemma 1.1.1]) shows us that $k(k-\lambda-1)=\mu(v-k-1)$.

Now let $u \in V(\Gamma)$. First we partition $V(\Gamma)$ into $V_{1}=\{u\}, V_{2}=\Gamma(u)$ and $V_{3}=$ $V(\Gamma) \backslash(\Gamma(u) \cup\{u\})$. Since each vertex in $V_{2}$ has $k-\lambda-1$ neighbours in $V_{3}$, there are $k(k-\lambda-1)$ edges between $V_{2}$ and $V_{3}$.

Define $b$ as the average number of neighbours a vertex in $V_{3}$ has in $V_{2}$. Then the number of edges between $V_{3}$ and $V_{2}$ is $b(v-k-1)$. Therefore, we have $k(k-\lambda-1)$ is equal to both $b(v-k-1)$ and $\mu(v-k-1)$, so $b=\mu$.

Let $w \in V_{3}$. The number of neighbours of $w$ in $V_{2}$ is at least $k-(v-k-2)=\mu$, as $\left|V_{3}\right|=v-k-1$. As $b=\mu$ is the average of numbers at least as big as $b$, they must all equal $b$. This means the number of common neighbours of $u$ and $w$ in $\Gamma$ is exactly $\mu$, and so $\Gamma$ is strongly regular. 


\subsection{The quintuple of parameters $(v, k, \lambda ; m, s)$}

Next we will give a necessary condition for the existence of a graph in $N G(v, k, \lambda ; m, s)$, in the form of an inequality that is linear in the parameters $k, \lambda, m, s$. When equality is achieved, we show that the parameters $(v, k, \lambda ; m, s)$ are extremal.

Firstly, we give a useful lemma involving Neumaier graphs where the neighbourhood of any vertex has a certain structure.

Lemma 3.2. Let $\Gamma$ be a graph from $N G(v, k, \lambda ; m, s)$. Further suppose that every vertex in $\Gamma$ has neighbourhood consisting of $l$ vertex disjoint cliques of size $s-1$. Then $\Gamma$ is strongly regular, with parameters $v=s+(l-1)(s-1) s / m, k=l(s-1), \lambda=(m-1)(l-1)+s-2$ and $\mu=l m$.

Proof. Take any vertex $u \in V(\Gamma)$ and $w \notin \Gamma(u)$. The neighbourhood of $u$ consists of disjoint $(s-1)$-cliques. Together with $u$ each of these cliques define an $s$-clique. These cliques are necessarily $m$-regular by Theorem 2.4. Thus $w$ is adjacent to $m$ vertices in each of these cliques, and has exactly $l m$ neighbours in common with $u$. This proves $\Gamma$ is strongly regular with $\mu=l m$.

The formulae for $k$ and $\lambda$ can be derived by simple counting arguments. Then for $v$, we use Proposition 2.5.

Now we give the inequality of the parameters $(v, k, \lambda)$ of a Neumaier graph. In the equality case, we show we are in a situation covered by Lemma 3.2, proving that $(v, k, \lambda ; m, s)$ is extremal.

Theorem 3.3. Let $\Gamma$ be a graph from $N G(v, k, \lambda ; m, s)$. Then

$$
k-\lambda-s+m-1 \geqslant 0
$$

Equality holds if and only if every vertex in $\Gamma$ has a neighbourhood consisting of two vertex disjoint $(s-1)$-cliques. In this case, $\Gamma$ is strongly regular with $v=s+(s(s-1) / m)$, $k=2(s-1), \lambda=s+m-3$ and $\mu=2 m$.

Proof. Let $S$ be an $m$-regular $s$-clique in $\Gamma$ and $u \in S$. Consider a vertex $w \in V(\Gamma) \backslash S$, with $u w \in E(\Gamma)$. We know that $u$ has $k-s$ other neighbours in $V(\Gamma) \backslash S$, and $w$ has $m-1$ neighbours in $S \backslash\{u\}$. Thus $u$ and $w$ have exactly $m-1$ common neighbours in $S$, and at most $k-s$ common neighbours in $V(\Gamma) \backslash S$. As $u, w$ have exactly $\lambda$ common neighbours, we must have $\lambda \leqslant k-s+m-1$.

When equality holds, we see that $w$ must be adjacent to all neighbours of $u$ in $V(\Gamma) \backslash S$. By repeating the argument for all other edges $u z$, with $z \in V(\Gamma) \backslash S$, we see that $u$ has a neighbourhood consisting of two vertex disjoint cliques.

By Proposition 2.5 (ii) and $k=\lambda+s-m+1$, we deduce that $(\lambda-s-m+3)(s-m)=0$. If $s=m, \Gamma$ is necessarily complete. Otherwise, $\lambda=s+m-3$ and $k=2(s-1)$. This proves that for all $u \in S, u$ has a neighbourhood consisting of two vertex disjoint $(s-1)$-cliques.

Now take a vertex $u \in V(\Gamma) \backslash S$. As $m \geqslant 1, u$ is adjacent to a vertex $w \in S$. As the neighbourhood of $w$ consists of $(s-1)$-cliques, $u$ is contained in $S^{\prime}$, which is one of these 
$(s-1)$-cliques. Then $S=S^{\prime} \cup\{w\}$ is an $s$-clique that contains $u$. Thus, we have proved that every vertex is contained in an $s$-clique.

By Theorem 2.4, any $s$-clique is necessarily $m$-regular. So we can apply the above argument to show that any vertex $u \in V(\Gamma)$ has a neighbourhood consisting of two vertex disjoint $(s-1)$-cliques in $\Gamma$. The result then follows from Lemma 3.2.

\subsubsection{Classifying the graphs in the equality case}

In fact, we can give a full description of all Neumaier graphs with parameters satisfying equality in $(*)$.

Theorem 3.4. Let $\Gamma$ be a graph from $N G(v, k, \lambda ; m, s)$, where $k-\lambda-s+m-1=0$. Then $\Gamma$ is one of the following strongly regular graphs:

(i) the square lattice graph $L_{2}(s)$;

(ii) the triangular graph $T(s+1)$, where $s \geqslant 3$;

(iii) the complete $s$-partite graph $K_{s \times 2}$, with parts of size 2 .

We prove this theorem by taking cases on the value of $m$. We start with the case $m=1$.

Lemma 3.5. Let $\Gamma$ be a graph from $N G(v, k, \lambda ; m, s)$, where $k-\lambda-s+m-1=0$ and $m=1$. Then $\Gamma$ is isomorphic to the square lattice graph $L_{2}(s)$.

Proof. By Theorem 3.3, $\Gamma$ is strongly regular with parameters $\left(s^{2}, 2(s-1), s-2,2\right)$. Any strongly regular graph with parameters $\left(s^{2}, 2(s-1), s-2,2\right)$ must be isomorphic to $L_{2}(s)$, unless $s=4$ (see [26]). In this case, there is only one strongly regular graph that is not isomorphic to $L_{2}(4)$, called the Shrikhande graph. This graph does not contain a regular clique (see [9]).

Next we consider the case $m=2$.

Lemma 3.6. Let $\Gamma$ be a graph from $N G(v, k, \lambda ; m, s)$, where $k-\lambda-s+m-1=0$ and $m=2$. Then $\Gamma$ is isomorphic to the triangular graph $T(s+1)$.

Proof. By Theorem 3.3, $\Gamma$ is strongly regular with parameters $\left(s^{2}, 2(s-1), s-2,2\right)$. Any strongly regular graph with parameters $\left(\left(\begin{array}{c}s+1 \\ 2\end{array}\right), 2(s-1), s-1,4\right)$ must be isomorphic to $T(s+1)$, unless $s=7$ (see $[10,20,25]$ or [9]). In this case, there are only three strongly regular graphs that are not isomorphic to $T(8)$, called the Chang graphs. Each of these does not contain a regular clique (see [9]).

Now we only need to consider the case $m \geqslant 3$. For this case, we can show that $m$ is particularly large with respect to $s$, which forces the graph to be isomorphic to $K_{s \times 2}$.

Lemma 3.7. Let $\Gamma$ be a graph from $N G(v, k, \lambda ; m, s)$, where $k-\lambda-s+m-1=0$ and $m \geqslant 3$. Then $\Gamma$ is isomorphic to $K_{s \times 2}$. 
Proof. We will first show that $m \geqslant 1+s / 2$.

Let $\Gamma$ be a graph in $N G(v, k, \lambda)$. Take a subset $S \subset V(\Gamma), S=\left\{u_{1}, u_{2}, \ldots, u_{s}\right\}$, where $S$ a $m$-regular $s$-clique in $\Gamma$. Without loss of generality, let $w \in V(\Gamma) \backslash S$, with $\left\{u_{1}, u_{2}, u_{3}\right\} \subseteq \Gamma(w) \cap S$. Note that by the equality case of Theorem 3.3, $w$ is adjacent to all neighbours of $u_{1}, u_{2}, u_{3}$ in $V(\Gamma) \backslash S$.

As $\Gamma$ is $k$-regular, we have $\left|N\left(u_{i}\right) \cap V(\Gamma) \backslash S\right|=k-s+1$ for all $i \in\{1, \ldots, s\}$. Also we must have $\left|N\left(u_{i}\right) \cap N\left(u_{j}\right) \cap V(\Gamma) \backslash S\right|=\lambda-s+2$ for all $i, j \in\{1, \ldots, s\}$. Thus we have

$$
\begin{aligned}
\left|\left(N\left(u_{1}\right) \cup N\left(u_{2}\right) \cup N\left(u_{3}\right)\right) \cap V(\Gamma) \backslash S\right|= & 3(k-s+1)-3(\lambda-s+2) \\
& +\left|\left(N\left(u_{1}\right) \cap N\left(u_{2}\right) \cap N\left(u_{3}\right)\right) \cap V(\Gamma) \backslash S\right|
\end{aligned}
$$

We see that $w$ is adjacent to at least $m+3(k-\lambda-1)$ vertices. Therefore, this has to be less than $k$. By using $\lambda=k-s+m-1$ and $k=2(s-1)$ (by Theorem 3.3), we have shown that $m \geqslant 1+s / 2$.

Let $u, w \in V(\Gamma) \backslash S$. As $S$ is $m$-regular and $m>s / 2$, there must exist a $i \in S$ such that $i u, i w \in E(\Gamma)$. We also know that the neighbourhood of $i$ in $V(\Gamma) \backslash S$ is a clique, so $u w \in E(\Gamma)$. Thus we have shown that $V(\Gamma) \backslash S$ is a clique in $\Gamma$.

By maximality of $S$, we must have $|V(\Gamma) \backslash S| \leqslant s$. Also, because $k=2(s-1)$, we must have $|V(\Gamma) \backslash S| \geqslant s-1$. As $\Gamma$ is non-complete, we have $|V(\Gamma) \backslash S|=s$, and $m=s-1$. By Theorem 3.3, $v=2 s, \lambda=2(s-2)$. Applying Proposition 3.1, we have shown the result.

\section{Small extremal parameter tuples and the smallest strictly Neumaier graph}

The following tables list all tuples $(v, k, \lambda, s, m)$ of integers, such that the following hold:

1. $0<k<v-1, v \leqslant 24,0 \leqslant \lambda<k, 2 \leqslant s \leqslant \lambda+2$ and $m \geqslant 1$.

2. 2 divides both $v k$ and $k \lambda$, and 6 divides $v k \lambda$ (see Lemma 2.3).

3. $C_{\tau}(m-1, s)=C_{\tau}(m, s)=0$, where $\tau=(v, k, \lambda)$ (see Lemma 2.4).

These tables were obtained by a straightforward computation using GAP [14]. All calculations were exact and took a total of about $20 \mathrm{CPU}$ milliseconds on a desktop PC.

Thus, if there is a Neumaier graph from $N G(v, k, \lambda ; m, s)$ such that $v \leqslant 24$, then the tuple $v, k, \lambda, m, s$ appears in our tables. The rightmost column of our tables display a result which proves that the tuple $(v, k, \lambda)$ or the tuple $(v, k, \lambda ; m, s)$ is extremal, or the symbol '-' otherwise. For example, L3.1 (i) refers to Lemma 3.1 part (i), and T3.3 refers to Theorem 3.3.

We see that Table 1 rules out all possible parameter tuples $(v, k, \lambda ; m, s)$ for a strictly Neumaier graph when $v<16$. Further, the table shows that any strictly Neumaier graph on 16 vertices is from $N G(16,9,4 ; 2,4)$. A graph in $N G(16,9,4 ; 2,4)$ is given in the next section. So Table 1 and this graph give the answer to Question A. 


\begin{tabular}{c|c|c|c|c|c}
$v$ & $k$ & $\lambda$ & $m$ & $s$ & result \\
\hline 4 & 2 & 0 & 1 & 2 & L3.1 (i) \\
\hline 6 & 3 & 0 & 1 & 2 & L3.1 (i) \\
\cline { 2 - 6 } & 4 & 2 & 2 & 3 & L3.1 (i) \\
\hline \multirow{3}{*}{8} & 4 & 0 & 1 & 2 & L3.1 (i) \\
\cline { 2 - 6 } & 6 & 4 & 3 & 4 & L3.1 (i) \\
\hline \multirow{4}{*}{9} & 4 & 1 & 1 & 3 & L2.7 \\
\cline { 2 - 6 } & 6 & 3 & 2 & 3 & L3.1 (i) \\
\hline \multirow{4}{*}{10} & 5 & 0 & 1 & 2 & L3.1 (i) \\
\cline { 2 - 6 } & 6 & 3 & 2 & 4 & L3.1 (iii) \\
\cline { 2 - 5 } & 8 & 6 & 4 & 5 & L3.1 (i) \\
\hline \multirow{5}{*}{12} & 5 & 2 & 1 & 4 & T3.3 \\
\cline { 2 - 5 } & 6 & 0 & 1 & 2 & L3.1 (i) \\
\cline { 2 - 5 } & 4 & 1 & 6 & T3.3 \\
\cline { 2 - 5 } & 8 & 4 & 2 & 3 & L3.1 (i) \\
\cline { 2 - 5 } & 9 & 6 & 3 & 4 & L3.1 (i) \\
\cline { 2 - 6 } & 10 & 8 & 5 & 6 & L3.1 (i)
\end{tabular}

\begin{tabular}{|c|c|c|c|c|c|}
\hline$v$ & $k$ & $\lambda$ & $m$ & $s$ & result \\
\hline \multirow[t]{3}{*}{14} & 7 & 0 & 1 & 2 & L3.1 (i) \\
\hline & 9 & 6 & 3 & 7 & T3.3 \\
\hline & 12 & 10 & 6 & 7 & L3.1 (i) \\
\hline \multirow[t]{6}{*}{15} & \multirow[t]{2}{*}{6} & 1 & 1 & 3 & L2.7 \\
\hline & & 3 & 1 & 5 & T3.3 \\
\hline & 8 & 4 & 2 & 5 & T3.3 \\
\hline & \multirow[t]{2}{*}{10} & 5 & 2 & 3 & L3.1 (i) \\
\hline & & 6 & 3 & 5 & Lii \\
\hline & 12 & 9 & 4 & 5 & L3.1 (i) \\
\hline \multirow[t]{7}{*}{16} & 6 & 2 & 1 & 4 & T3.3 \\
\hline & \multirow[t]{2}{*}{8} & 0 & 1 & 2 & L3.1 (i) \\
\hline & & 6 & 1 & 8 & T3.3 \\
\hline & 9 & 4 & 2 & 4 & - \\
\hline & 10 & 6 & 3 & 6 & L3.1 (iii) \\
\hline & 12 & 8 & 3 & 4 & L3.1 (i) \\
\hline & 14 & 12 & 7 & 8 & L3.1 (i) \\
\hline
\end{tabular}

Table 1. Possible parameters of Neumaier graphs on $v \leqslant 16$ vertices

Table 1 and 2 together show that $(24,8,2)$ is the only possible parameter tuple for a strictly Neumaier graph containing a 1-regular clique when $v \leqslant 24$.

Finally, direct computations can show that there is only one strictly Neumaier graph in $N G(16,9,4 ; 2,4)$, up to isomorphism, and there are no strictly Neumaier graphs with parameter tuples $(21,14,9 ; 4,7)$ and $(22,12,5 ; 2,4)$. Let us explain some ideas of the computations. We fix a subgraph induced by vertices of a clique with given size. Then we exhaust all regular graphs such that the fixed clique is regular with given nexus. Using MAGMA [2], we find that the graphs in $N G(16,9,4 ; 2,4)$ are isomorphic pairwise.

Thus we have found that any strictly Neumaier graph on at most 24 vertices must have parameters $(16,9,4 ; 2,4)$ or $(24,8,2 ; 1,4)$.

\subsection{Vertex-transitive strictly Neumaier graphs}

The authors discovered the smallest strictly Neumaier graph independently, using completely different approaches.

Goryainov and Panasenko were looking for strictly Neumaier graphs that admit a partition into regular cliques and used this pattern for computer searching.

Evans found the graph in a collection of vertex-transitive edge-regular graphs received from Gordon Royle. Holt and Royle have recently enumerated all transitive permutation groups of degree at most 47 [21]. From this, Royle was able to enumerate all vertextransitive edge-regular graphs on less than 47 vertices.

Thus we also find all vertex-transitive strictly Neumaier graphs on at most 47 vertices 


\begin{tabular}{|c|c|c|c|c|c|}
\hline$v$ & $k$ & $\lambda$ & $m$ & $s$ & result \\
\hline \multirow[t]{5}{*}{18} & 7 & 4 & 1 & 6 & T3.3 \\
\hline & 9 & 0 & 1 & 2 & L3.1 (i) \\
\hline & 12 & 6 & 2 & 3 & L3.1 (i) \\
\hline & 15 & 12 & 5 & 6 & L3.1 (i) \\
\hline & 16 & 14 & 8 & 9 & L3.1 (i) \\
\hline \multirow[t]{4}{*}{20} & 10 & 0 & 1 & 2 & L3.1 (i) \\
\hline & 15 & 10 & 3 & 4 & L3.1 (i) \\
\hline & 16 & 12 & 4 & 5 & L3.1 (i) \\
\hline & 18 & 16 & 9 & 10 & L3.1 (i) \\
\hline \multirow[t]{10}{*}{21} & \multirow[t]{2}{*}{8} & 1 & 1 & 3 & $\mathrm{~L} 2.7$ \\
\hline & & 5 & 1 & 7 & T3.3 \\
\hline & 10 & 5 & 2 & 6 & T3.3 \\
\hline & \multirow[t]{2}{*}{12} & 7 & 3 & 7 & T3.3 \\
\hline & & 8 & 3 & 9 & T3.3 \\
\hline & \multirow[t]{2}{*}{14} & 7 & 2 & 3 & L3.1 (i) \\
\hline & & 9 & 4 & 7 & - \\
\hline & 15 & 10 & 4 & 6 & L3.1 (ii) \\
\hline & 16 & 12 & 6 & 9 & L3.1 (ii) \\
\hline & 18 & 15 & 6 & 7 & L3.1 (i) \\
\hline
\end{tabular}

\begin{tabular}{|c|c|c|c|c|c|}
\hline$v$ & $k$ & $\lambda$ & $m$ & $s$ & result \\
\hline \multirow[t]{5}{*}{22} & 11 & 0 & 1 & 2 & L3.1 (i) \\
\hline & 12 & 5 & 2 & 4 & - \\
\hline & 14 & 9 & 4 & 8 & T3.3 \\
\hline & 16 & 12 & 6 & 11 & T3.3 \\
\hline & 20 & 18 & 10 & 11 & L3.1 (i) \\
\hline \multirow[t]{12}{*}{24} & \multirow[t]{2}{*}{8} & 2 & 1 & 4 & - \\
\hline & & 4 & 1 & 6 & T3.3 \\
\hline & 9 & 6 & 1 & 8 & T3.3 \\
\hline & \multirow[t]{2}{*}{12} & 0 & 1 & 2 & L3.1 (i) \\
\hline & & 10 & 1 & 12 & T3.3 \\
\hline & 16 & 8 & 2 & 3 & L3.1 (i) \\
\hline & \multirow[t]{2}{*}{18} & 12 & 3 & 4 & L3.1 (i) \\
\hline & & 15 & 6 & 16 & T3.3 \\
\hline & \multirow[t]{2}{*}{20} & 16 & 5 & 6 & L3.1 (i) \\
\hline & & 17 & 10 & 16 & T3.3 \\
\hline & 21 & 18 & 7 & 8 & L3.1 (i) \\
\hline & 22 & 20 & 11 & 12 & L3.1 (i) \\
\hline
\end{tabular}

Table 2. Possible parameters of Neumaier graphs on $16<v \leqslant 24$ vertices

using the enumeration [21]. We list the parameters of all vertex-transitive strictly Neumaier graph on at most 47 vertices, and the number of vertex-transitive strictly Neumaier graphs with these parameters.

(i) 1 graph with parameters $(16,9,4 ; 2,4)$.

(ii) 4 graphs with parameters $(24,8,2 ; 1,4)$.

(iii) 2 graphs with parameters $(28,9,2 ; 1,4)$.

(iv) 1 graph with parameters $(40,12,2 ; 1,4)$.

We note that the four vertex-transitive strictly Neumaier graphs in $N G(24,8,2 ; 1,4)$ appear in [17]. They come about in a search for Deza graphs, which are a certain generalisation of strongly regular graphs.

\section{Two constructions and two generalisations of the smallest strictly Neumaier graph}

In this section, we will construct two sequences of strictly Neumaier graphs that generalise the smallest strictly Neumaier graph. The motivation behind both constructions is as follows. 
Consider a graph $\Gamma$, and two disjoint subsets $S, T$ of the vertices of $\Gamma$. Now we introduce an important operation on the graph $\Gamma$. For each vertex $v$ in $S$, we do the following. First take $N=N(v) \cap T$, the neighbours of $v$ in $T$, and $M=T \backslash N$. Then delete all edges $v u$ where $u$ is in $N$, and insert all edges $v u$ where $u$ is in $M$. We will call this operation a switching of the edges between $S$ and $T$ in the graph $\Gamma$.

Note that the smallest Neumaier graph contains disjoint 2-regular 4-cliques. A switching between any distinct pair of these cliques will not change the fact that they are 2regular. Therefore, if we could find a strongly regular graph with these parameters and containing disjoint 2-regular 4-cliques, we could hope that the smallest Neumaier graph is the result of switching edges between them.

In the following subsections we will see that the smallest Neumaier graph is the result of two consecutive switchings of the graph $\mathrm{VO}^{+}(4,2)$. We then generalise our switchings to the graphs $\mathrm{VO}^{+}(2 e, 2)$ for larger $e$, and construct infinite sequences of strictly Neumaier graphs with the same edge-regular parameters as $\operatorname{VO}^{+}(2 e, 2)$. From now on, we will denote $\mathrm{VO}^{+}(2 e, 2)$ as the graph $\Gamma_{e}$. Throughout this section we use matrix notation with stars ' $*$ ' as entries, which denotes the set of corresponding matrices where the stars take all possible values independently.

\subsection{The first construction of the smallest Neumaier graph}

Consider the 1-dimensional subspace

$$
W=\left(\begin{array}{cc}
* & 0 \\
0 & 0
\end{array}\right)
$$

According to Lemma 2.8, the subspace $W$ is contained in exactly two generators. These are the spaces

$$
W_{1}=\left(\begin{array}{cc}
* & * \\
0 & 0
\end{array}\right) \text { and } W_{2}=\left(\begin{array}{cc}
* & 0 \\
0 & *
\end{array}\right)
$$

Take the vector

$$
v=\left(\begin{array}{ll}
0 & 0 \\
1 & 0
\end{array}\right)
$$

and consider the cosets

$$
\begin{aligned}
& v+W_{1}=\left(\begin{array}{ll}
* & * \\
1 & 0
\end{array}\right), \\
& v+W_{2}=\left(\begin{array}{ll}
* & 0 \\
1 & *
\end{array}\right),
\end{aligned}
$$

whose intersection is

$$
v+W=\left(\begin{array}{cc}
* & 0 \\
1 & 0
\end{array}\right)
$$

In this setting, the adjacency matrix of the affine polar graph $\Gamma_{2}=V O^{+}(4,2)$ can be seen in Figure 1. The graph $\Gamma_{2}$ is isomorphic to the complement of the square lattice graph $L_{2}(4)$. 
We note that switching edges between the cliques $W_{1}, v+W_{1}$ gives a graph isomorphic to the complement of the Shrikhande graph. The switching of edges between the cliques $W_{1}, v+W_{1}$ and then between the cliques $W_{2}, v+W_{2}$ is equivalent to inverting the highlighted entries in Figure 1. This gives the strictly Neumaier graph $\Gamma_{2,1}$ on 16 vertices, whose adjacency matrix is presented in Figure 2. The notation in the rightmost column of Figure 2 means the following. Two rows have the same letter if and only if they correspond to non-adjacent vertices having 8 common neighbours; two rows have the same number if and only if they correspond to non-adjacent vertices having 4 common neighbours. Otherwise, every two non-adjacent vertices have 6 common neighbours; every two adjacent vertices have 4 common neighbours.

\subsection{The first generalisation of the smallest strictly Neumaier graph}

In this subsection we generalise the construction from Subsection 5.1.

Take the $(e-1)$-dimensional subspace

$$
W=\left(\begin{array}{ccc|cc}
* & \ldots & * & * & 0 \\
0 & \ldots & 0 & 0 & 0
\end{array}\right)
$$

where the size of matrices is $2 \times e$. According to Lemma 2.8, the subspace $W$ is contained in exactly two generators. These are the spaces

$$
W_{1}=\left(\begin{array}{ccc|cc}
* & \ldots & * & * & * \\
0 & \ldots & 0 & 0 & 0
\end{array}\right) \text { and } W_{2}=\left(\begin{array}{ccc|cc}
* & \ldots & * & * & 0 \\
0 & \ldots & 0 & 0 & *
\end{array}\right) \text {. }
$$

Take the vector

$$
v=\left(\begin{array}{lll|ll}
0 & \ldots & 0 & 0 & 0 \\
0 & \ldots & 0 & 1 & 0
\end{array}\right)
$$

and consider the cosets

$$
v+W_{1}=\left(\begin{array}{ccc|cc}
* & \ldots & * & * & * \\
0 & \ldots & 0 & 1 & 0
\end{array}\right), \quad v+W_{2}=\left(\begin{array}{ccc|cc}
* & \ldots & * & * & 0 \\
0 & \ldots & 0 & 1 & *
\end{array}\right)
$$

whose intersection is

$$
v+W=\left(\begin{array}{ccc|cc}
* & \ldots & * & * & 0 \\
0 & \ldots & 0 & 1 & 0
\end{array}\right) .
$$

Denote by $\Gamma_{e, 1}=\Gamma_{e}\left(W, W_{1}, W_{2}, v\right)$ the graph obtained from $\Gamma_{e}=V O^{+}(2 e, 2)$ by switching edges between the cliques $W_{1}, v+W_{1}$ and then between the cliques $W_{2}, v+W_{2}$. Let $(n, k, \lambda, \mu)$ be the parameters of the affine polar graph $\Gamma_{e}=V^{+}(2 e, 2)$ as a strongly regular graph.

Theorem 5.1. The graph $\Gamma_{e, 1}$ is a strictly Neumaier graph with parameters

$$
\left(2^{2 e},\left(2^{e-1}+1\right)\left(2^{e}-1\right), 2\left(2^{e-2}+1\right)\left(2^{e-1}-1\right) ; 2^{e-1}, 2^{e}\right) .
$$

Further, the number of common neighbours of two non-adjacent vertices in the graph takes the values $\mu-2^{e-1}, \mu$ and $\mu+2^{e-1}$. 


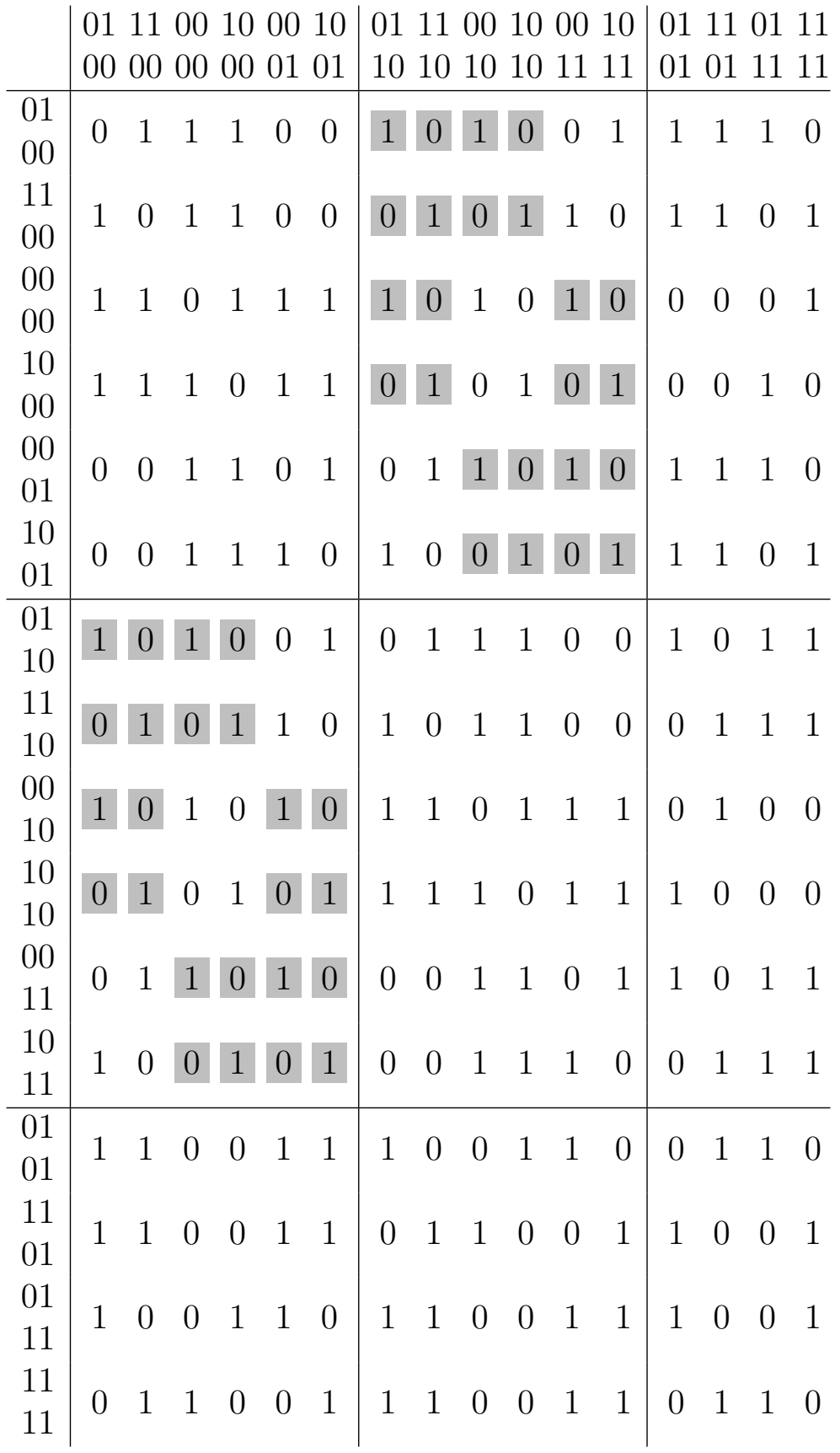

Figure 1. The adjacency matrix, $A_{2}$, of $\Gamma_{2}=V O^{+}(4,2)$

Proof. For any $a, b, c, d \in \mathbb{F}_{2}$, let

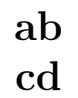

denote the set of matrices

$$
\left(\begin{array}{lll|ll}
* & \ldots & * & a & b \\
0 & \ldots & 0 & c & d
\end{array}\right)
$$




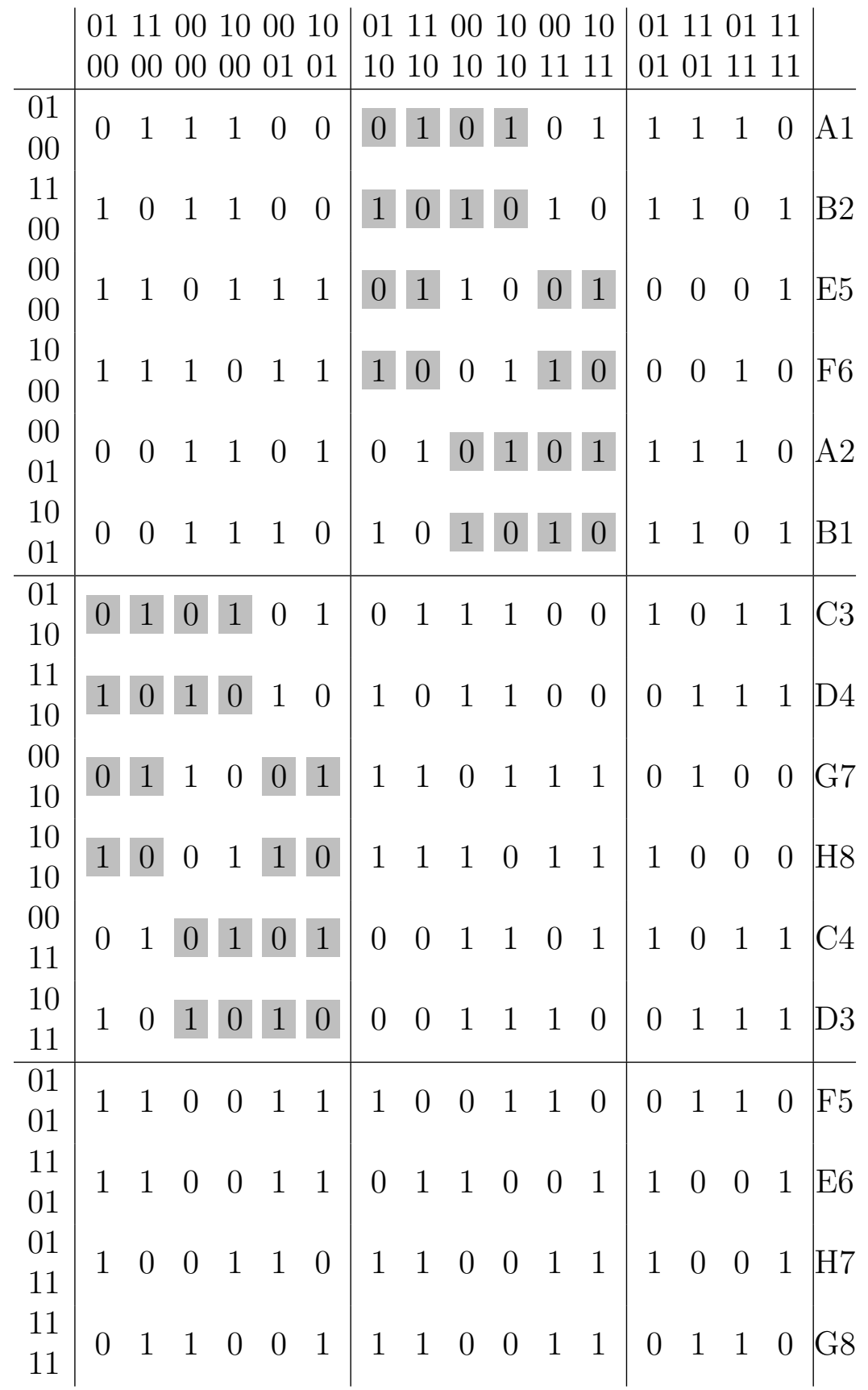

Figure 2. The adjacency matrix, $A_{2,1}$, of the graph $\Gamma_{2,1}$

Consider the subgraph $\Delta$ of $\Gamma_{e}=V O^{+}(2 e, 2)$ induced by the set of all matrices

$$
\left(\begin{array}{lll|ll}
* & \ldots & * & a & b \\
0 & \ldots & 0 & c & d
\end{array}\right)
$$

where $a, b, c, d$ run over $\mathbb{F}_{2}$. The adjacency matrix of the subgraph $\Delta$ is presented by the block-matrix in Figure 3 , where $K$ denotes the adjacency matrix of the complete 
graph on $2^{e-2}$ vertices; $J$ denotes the all-ones matrix of size $2^{e-2} \times 2^{e-2} ; Z$ denotes the all-zeroes matrix of size $2^{e-2} \times 2^{e-2}$.

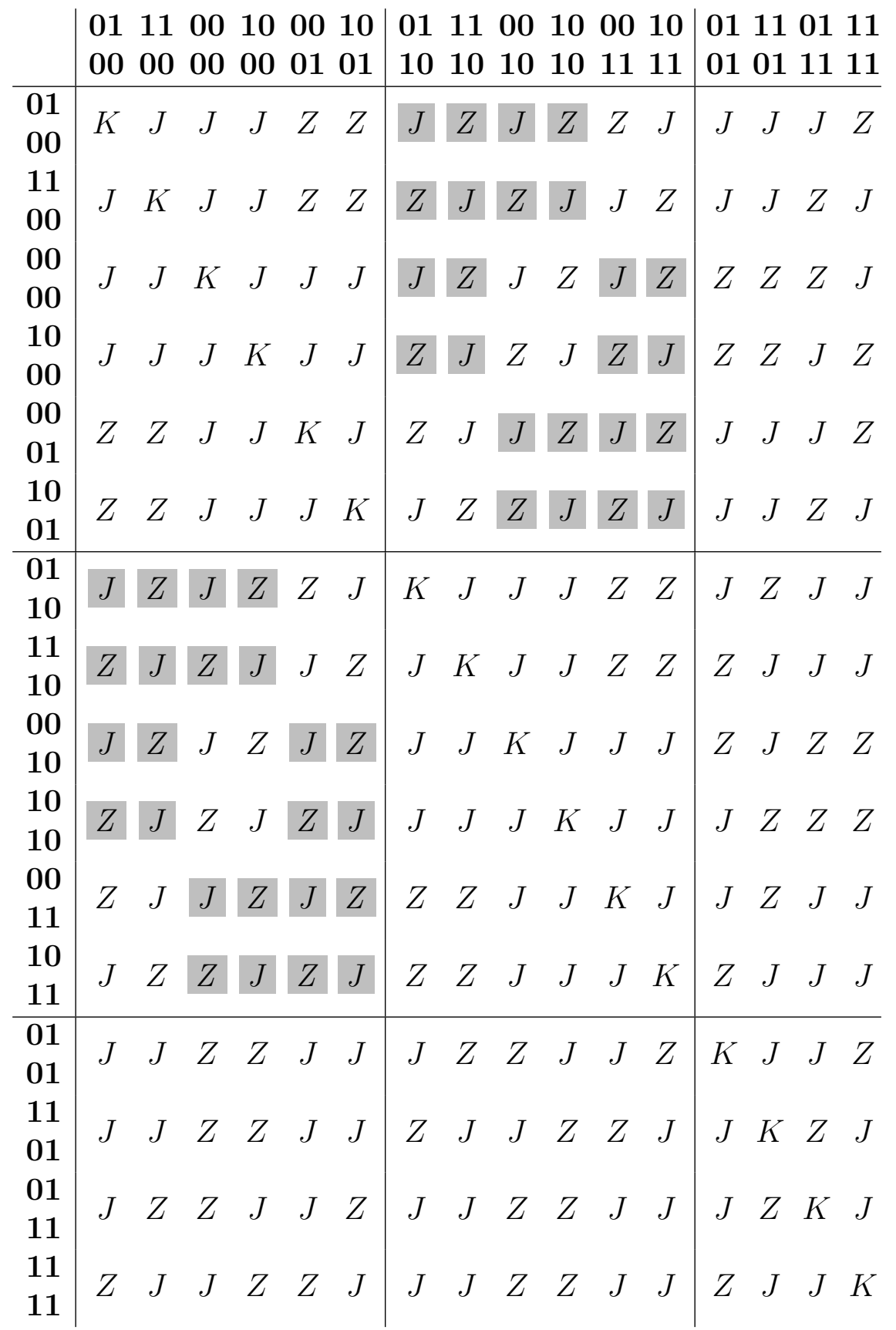

Figure 3. The adjacency matrix, $A_{e}$, of the subgraph $\Delta$ of $\Gamma_{e}=V O^{+}(2 e, 2)$

We invert a block of the matrix by inverting each entry of the block. Therefore, inverting a block with entry $J$ results in a block with entry $Z$, and inverting a block with entry $Z$ results in a block with entry $J$. Switching edges between the cliques $W_{1}, v+W_{1}$ and then between the cliques $W_{2}, v+W_{2}$ is equivalent to inverting the highlighted entries 
in Figure 3. This gives the submatrix of the adjacency matrix of $\Gamma_{e, 1}=\Gamma\left(W, W_{1}, W_{2}, v\right)$ presented in Figure 4. Note that every switched edge connects vertices from the subgraph $\Delta$. This means that the switching preserves all edges having a vertex outside of $\Delta$.

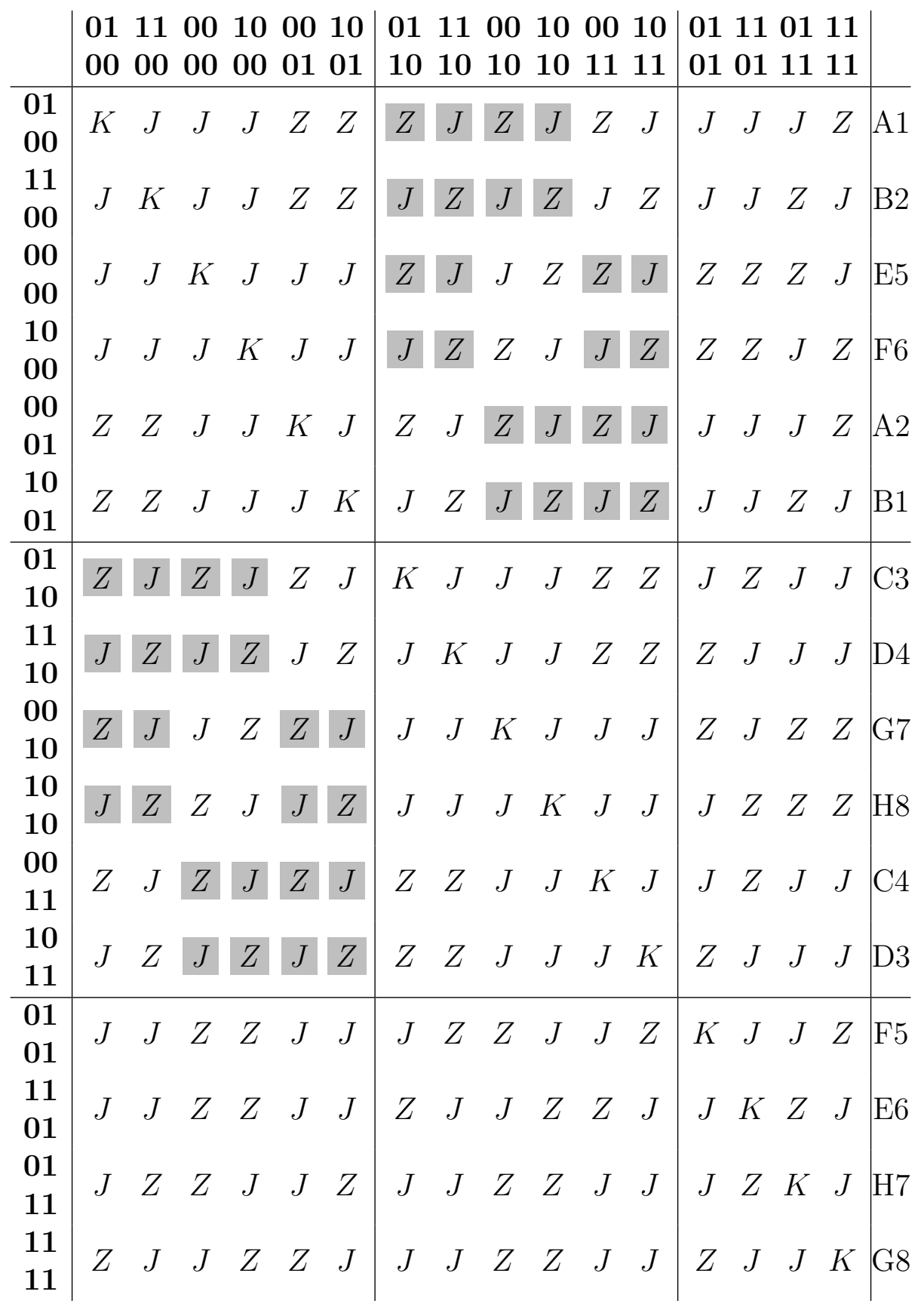

Figure 4. The adjacency matrix, $A_{e, 1}$, of the subgraph $\Delta$ of $\Gamma_{e, 1}=\Gamma_{e}\left(W, W_{1}, W_{2}, v\right)$

Let $(n, k, \lambda, \mu)$ be the parameters of the affine polar graph $\Gamma_{e}=V O^{+}(2 e, 2)$ as a strongly regular graph. We have to check that the obtained graph is a strictly Neumaier 
graph. Note that $W_{1}$ is a regular clique in $\Gamma_{e, 1}=\Gamma\left(W, W_{1}, W_{2}, v\right)$. Let us check that any pair of vertices in $\Gamma_{e, 1}$ is OK, i.e., any two adjacent vertices have $\lambda$ common neighbours. Also, we investigate which values of $\mu$ occur in $\Gamma_{e, 1}$.

Let us consider any two vertices inside of $\Delta$. The notation in the right column of the matrix in Figure 4 means the following. Two block-rows have the same letter if and only if any row from the one block-row and any row from the other block-row correspond to non-adjacent vertices having $\mu+2^{e-1}$ common neighbours; two block-rows have the same number if and only if any row from the one block-row and any row from the other blockrow correspond to non-adjacent vertices having $\mu-2^{e-1}$ common neighbours. Otherwise, every two non-adjacent vertices corresponding to rows of this submatrix have $\mu$ common neighbours; every two adjacent vertices have $\lambda$ common neighbours. This means that all pairs of vertices inside of $\Delta$ are OK.

Let us consider any two vertices outside of $\Delta$. Their neighbours and, consequently, their common neighbours are preserved by the switching. This means that all pairs of vertices outside of $\Delta$ are OK.

Let us consider a vertex $x$ in $\Delta$ and a vertex $y$ outside of $\Delta$. If the neighbours of $x$ are preserved by the switching, then $x, y$ are OK. Assume that the neighbours of $x$ are switched. Then the vertices $x, y$ are OK since the vertex $y$ is adjacent to half the of vertices of each block of $\Delta$. In fact, the vertex $y$ is given by a matrix

$$
\left(\begin{array}{ccc|cc}
y_{1} & \cdots & y_{2 e-5} & y_{2 e-3} & y_{2 e-1} \\
y_{2} & \cdots & y_{2 e-4} & y_{2 e-2} & y_{2 e}
\end{array}\right)
$$

where there is at least one non-zero among $y_{2}, y_{4}, \ldots, y_{2 e-4}$. Without loss of generality, assume that $y_{2}=1$. Let us show that $y$ is adjacent to half of the vertices in a block

$$
\left(\begin{array}{lll|ll}
* & \ldots & * & a & b \\
0 & \ldots & 0 & c & d
\end{array}\right) \text {. }
$$

We have

$$
\begin{aligned}
y+\left(\begin{array}{ccc|cc}
* & \ldots & * & a & b \\
0 & \ldots & 0 & c & d
\end{array}\right) & =\left(\begin{array}{ccc|cc}
* & \ldots & * & a^{\prime} & b^{\prime} \\
1 & \ldots & y_{2 e-4} & c^{\prime} & d^{\prime}
\end{array}\right) \\
& =\left(\begin{array}{ccc|cc}
0 & \ldots & * & a^{\prime} & b^{\prime} \\
1 & \ldots & y_{2 e-4} & c^{\prime} & d^{\prime}
\end{array}\right) \bigcup\left(\begin{array}{ccc|cc}
1 & \ldots & * & a^{\prime} & b^{\prime} \\
1 & \ldots & y_{2 e-4} & c^{\prime} & d^{\prime}
\end{array}\right) \\
& =Y_{0} \cup Y_{1}
\end{aligned}
$$

Note that $\left|Y_{0}\right|=\left|Y_{1}\right|$, and the form $Q$ has value 0 on one of the sets $Y_{0}, Y_{1}$ and value 1 on the other. We have proved that the switching preserves the number of common neighbours $x$ and $y$, completing the proof of the theorem.

\subsection{The second construction of the smallest strictly Neumaier graph}

Consider the graph $\Gamma_{2}=\operatorname{VO}^{+}(4,2)$. Take the generator

$$
W_{1}=\left(\begin{array}{cc}
* & * \\
0 & 0
\end{array}\right)
$$


the vector

$$
v=\left(\begin{array}{ll}
0 & 0 \\
0 & 1
\end{array}\right)
$$

and the coset

$$
v+W_{1}=\left(\begin{array}{cc}
* & * \\
0 & 1
\end{array}\right) .
$$

Divide vertices of the 2-regular 4-cliques $W_{1}$ and $v+W_{1}$ into two parts as

$$
\begin{gathered}
W_{1}=V_{0} \cup V_{1}, \\
v+W_{1}=V_{2} \cup V_{3},
\end{gathered}
$$

where

$$
\begin{aligned}
& V_{0}=\left(\begin{array}{ll}
* & 0 \\
0 & 0
\end{array}\right), \\
& V_{1}=\left(\begin{array}{ll}
* & 1 \\
0 & 0
\end{array}\right), \\
& V_{2}=\left(\begin{array}{ll}
* & 0 \\
0 & 1
\end{array}\right), \\
& V_{3}=\left(\begin{array}{ll}
* & 1 \\
0 & 1
\end{array}\right) .
\end{aligned}
$$

Note that there are all possible edges between $V_{0}$ and $V_{2}$, there are all possible edges between $V_{1}$ and $V_{3}$, there are no edges between $V_{0}$ and $V_{3}$, and there are no edges between $V_{1}$ and $V_{2}$. Denote by $\Gamma_{2}^{\prime}$ the graph obtained from $\Gamma_{2}$ by switching edges between the cliques $W_{1}$ and $v+W_{1}$. Note that each of the sets $V_{0} \cup V_{3}$ and $V_{1} \cup V_{2}$ induces a 4-clique in $\Gamma_{2}^{\prime}$.

The set

$$
C:=\left(\begin{array}{cc}
* & 0 \\
1 & *
\end{array}\right)
$$

induces a 2-regular 4-clique in the graph $\Gamma_{2}^{\prime}$ as well as in $\Gamma_{2}$ since the switching between $W_{1}$ and $v+W_{1}$ did not modify the neighbourhoods of the vertices from $C$. Moreover, $C \cap\left(W_{1} \cup v+W_{1}\right)=\varnothing$ holds, and any vertex from $C$ is adjacent to half of the vertices of each of the sets $V_{0}, V_{1}, V_{2}, V_{3}$. This means that the switching between the cliques $V_{1} \cup V_{2}, C$ and the switching between the cliques $V_{0} \cup V_{3}, C$ preserve the regularity of $\Gamma_{2}^{\prime}$. Denote by $\Gamma_{2}^{\prime \prime}$ and $\Gamma_{2}^{\prime \prime \prime}$ the graphs obtained from $\Gamma_{2}^{\prime}$ by applying these two switchings, respectively. One can prove that the graphs $\Gamma_{2}^{\prime \prime}$ and $\Gamma_{2}^{\prime \prime \prime}$ are isomorphic to the smallest Neumaier graph. Now we show how can the adjacency matrix of the graph $\Gamma_{2}^{\prime \prime}$ be obtained from the adjacency matrix of $\Gamma_{2}$.

In this setting, the adjacency matrix of the affine polar graph $\Gamma_{2}=\mathrm{VO}^{+}(4,2)$ can be written as in Figure 5.

Switching edges between the cliques $W_{1}, v+W_{1}$ and then between the cliques $V_{1} \cup V_{2}$, $C$ is equivalent to inverting the highlighted entries in Figure 5. This gives the strictly Neumaier graph $\Gamma_{2,2}$ on 16 vertices, whose adjacency matrix is presented in Figure 6. 


\begin{tabular}{|c|c|c|c|c|c|c|c|c|c|c|c|c|c|}
\hline & $\begin{array}{l}10 \\
00 \\
0\end{array}$ & $\begin{array}{ll}1 & 11 \\
0 & 00\end{array}$ & $\begin{array}{ll}00 & 10 \\
01 & 01 \\
\end{array}$ & 01 & & $\begin{array}{l}00 \\
10\end{array}$ & & 11 & 11 & 10 & & & \\
\hline $\begin{array}{l}00 \\
00\end{array}$ & $\begin{array}{ll}0 & 1\end{array}$ & $\begin{array}{ll}1 & 1\end{array}$ & $\begin{array}{ll}1 & 1\end{array}$ & 0 & & 1 & 0 & 1 & 0 & 1 & 0 & & \\
\hline 0 & 10 & 11 & $\begin{array}{ll}1 & 1\end{array}$ & 0 & & 0 & 1 & 0 & 1 & 0 & 1 & 1 & \\
\hline 00 & $\begin{array}{ll}1 & 1\end{array}$ & $\begin{array}{ll}0 & 1\end{array}$ & $\begin{array}{ll}0 & 0\end{array}$ & 1 & & 1 & 0 & 0 & 1 & 1 & 0 & & \\
\hline 00 & $\begin{array}{ll}1 & 1\end{array}$ & 10 & $\begin{array}{ll}0 & 0\end{array}$ & 1 & 1 & 0 & 1 & 1 & 0 & 0 & 1 & 0 & \\
\hline 01 & \begin{tabular}{l|l}
1 & 1
\end{tabular} & 0 & & 1 & & 1 & 0 & 1 & 0 & 0 & 1 & 1 & 0 \\
\hline 01 & $\begin{array}{ll}1 & 1\end{array}$ & & & 1 & 1 & 0 & 1 & 0 & 1 & 1 & 0 & 0 & 1 \\
\hline $\begin{array}{l}01 \\
01\end{array}$ & 0 & 1 & & 0 & 1 & 0 & 1 & 1 & 0 & 1 & 0 & 1 & 0 \\
\hline 01 & 0 & & 11 & & 0 & 1 & 0 & 0 & 1 & 0 & 1 & 0 & 1 \\
\hline 10 & $\begin{array}{ll}1 & 0\end{array}$ & $\begin{array}{ll}1 & 0\end{array}$ & $\begin{array}{ll}1 & 0\end{array}$ & & 1 & 0 & 1 & 1 & 1 & 1 & 1 & 0 & 0 \\
\hline 10 & $\begin{array}{ll}0 & 1\end{array}$ & $\begin{array}{lll}0 & 1\end{array}$ & $\begin{array}{ll}0 & 1\end{array}$ & 1 & 0 & 1 & 0 & 1 & 1 & 1 & 1 & 0 & 0 \\
\hline 11 & 10 & $\begin{array}{lll}0 & 1\end{array}$ & $\begin{array}{ll}1 & 0\end{array}$ & & 0 & 1 & 1 & 0 & 1 & 0 & 0 & 1 & 1 \\
\hline 11 & $\begin{array}{ll}0 & 1\end{array}$ & 10 & $\begin{array}{ll}0 & 1\end{array}$ & 0 & 1 & 1 & 1 & 1 & 0 & 0 & 0 & 1 & 1 \\
\hline 1 & $\begin{array}{ll}1 & 0\end{array}$ & 10 & & 1 & 0 & 1 & 1 & 0 & 0 & 0 & 1 & 1 & \\
\hline 11 & $\begin{array}{ll}0 & 1\end{array}$ & $\begin{array}{ll}0 & 1\end{array}$ & $\begin{array}{ll}1 & 0\end{array}$ & & 1 & 1 & 1 & 0 & 0 & 1 & 0 & 1 & \\
\hline 01 & $\begin{array}{ll}0 & 1\end{array}$ & 10 & $\begin{array}{ll}1 & 0\end{array}$ & & 0 & 0 & 0 & 1 & 1 & 1 & 1 & 0 & \\
\hline 11 & $\begin{array}{ll}1 & 0\end{array}$ & $\begin{array}{ll}0 & 1\end{array}$ & $\begin{array}{ll}0 & 1\end{array}$ & & 1 & 0 & 0 & 1 & 1 & 1 & 1 & 1 & 0 \\
\hline
\end{tabular}

Figure 5. The adjacency matrix, $B_{2}$, of $\Gamma_{2}$

The notation in the rightmost column of Figure 6 means the following. Two rows have the same letter if and only if they correspond to non-adjacent vertices having 8 common neighbours; two rows have the same number if and only if they correspond to non-adjacent vertices having 4 common neighbours. Otherwise, every two non-adjacent vertices have 6 common neighbours; every two adjacent vertices have 4 common neighbours. 


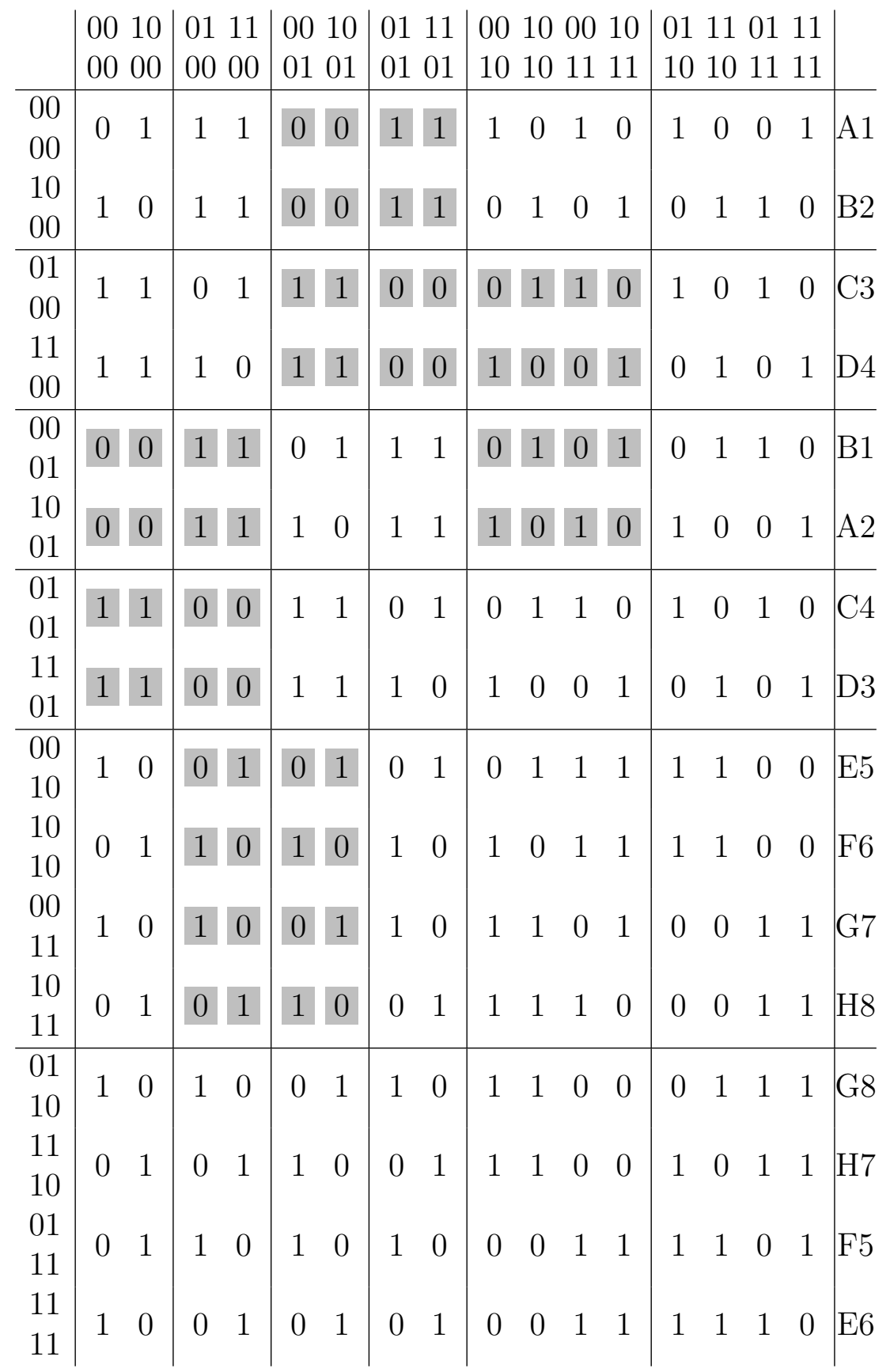

Figure 6. The adjacency matrix, $B_{2,2}$, of $\Gamma_{2,2}$

\subsection{The second generalisation of the smallest strictly Neumaier graph}

In this subsection we generalise the construction from Subsection 5.3 and present one more family of strictly Neumaier graphs.

For any $e \geqslant 2$, consider the affine polar graph $\Gamma_{e}=\operatorname{VO}^{+}(2 e, 2)$ and take the regular 
clique given by the generator

$$
W_{1}=\left(\begin{array}{ccc|cc}
* & \ldots & * & * & * \\
0 & \ldots & 0 & 0 & 0
\end{array}\right)
$$

For the vector

$$
v=\left(\begin{array}{lll|ll}
0 & \ldots & 0 & 0 & 0 \\
0 & \ldots & 0 & 0 & 1
\end{array}\right)
$$

take the regular clique

$$
v+W_{1}=\left(\begin{array}{ccc|cc}
* & \ldots & * & * & * \\
0 & \ldots & 0 & 0 & 1
\end{array}\right)
$$

which lies in the spread given by $W_{1}$. Divide $W_{1}$ and $v+W_{1}$ into two parts as

$$
\begin{gathered}
W_{1}=V_{0} \cup V_{1}, \\
v+W_{1}=V_{2} \cup V_{3},
\end{gathered}
$$

where

$$
\begin{aligned}
V_{0} & =\left(\begin{array}{lll|ll}
* & \ldots & * & * & 0 \\
0 & \ldots & 0 & 0 & 0
\end{array}\right), \\
V_{1} & =\left(\begin{array}{lll|ll}
* & \ldots & * & * & 1 \\
0 & \ldots & 0 & 0 & 0
\end{array}\right), \\
V_{2} & =\left(\begin{array}{lll|ll}
* & \ldots & * & * & 0 \\
0 & \ldots & 0 & 0 & 1
\end{array}\right), \\
V_{3} & =\left(\begin{array}{lll|ll}
* & \ldots & * & * & 1 \\
0 & \ldots & 0 & 0 & 1
\end{array}\right) .
\end{aligned}
$$

Note that there are all possible edges between $V_{0}$ and $V_{2}$, there are all possible edges between $V_{1}$ and $V_{3}$, there are no edges between $V_{0}$ and $V_{3}$, and there are no edges between $V_{1}$ and $V_{2}$. Denote by $\Gamma_{e}^{\prime}$ the graph obtained from $\Gamma_{e}$ by switching edges between the cliques $W_{1}$ and $v+W_{1}$. Note that each of the sets $V_{0} \cup V_{3}$ and $V_{1} \cup V_{2}$ induces a $2^{e}$-clique in $\Gamma_{e}^{\prime}$.

The set

$$
C:=\left(\begin{array}{ccc|cc}
* & \ldots & * & * & 0 \\
0 & \ldots & 0 & 1 & *
\end{array}\right)
$$

induces a $2^{e-1}$-regular $2^{e}$-clique in the graph $\Gamma_{e}^{\prime}$ as well as in $\Gamma_{e}$ since the switching between $W_{1}$ and $v+W_{1}$ did not modify the neighbourhoods of the vertices from $C$. Moreover, $C \cap\left(W_{1} \cup v+W_{1}\right)=\varnothing$ holds, and any vertex from $C$ is adjacent to half of the vertices of each of the sets $V_{0}, V_{1}, V_{2}, V_{3}$. This means that the switching between the cliques $V_{1} \cup V_{2}$, $C$ and the switching between the cliques $V_{0} \cup V_{3}, C$ preserve the regularity of $\Gamma_{e}^{\prime}$. Denote by $\Gamma_{e, 2}$ the graph obtained from $\Gamma_{e}^{\prime}$ by switching edges between the cliques $W_{1} \cup W_{2}$ and $C$. Let $(n, k, \lambda, \mu)$ be the parameters of the affine polar graph $\Gamma_{e}=\operatorname{VO}^{+}(2 e, 2)$ as a strongly regular graph. 
Theorem 5.2. The graph $\Gamma_{e, 2}$ is a strictly Neumaier graph with parameters

$$
\left(2^{2 e},\left(2^{e-1}+1\right)\left(2^{e}-1\right), 2\left(2^{e-2}+1\right)\left(2^{e-1}-1\right) ; 2^{e-1}, 2^{e}\right) .
$$

Further, the number of common neighbours of two non-adjacent vertices in the graph takes the values $\mu-2^{e-1}, \mu$ and $\mu+2^{e-1}$.

Proof. For any $a, b, c, d \in \mathbb{F}_{2}$, let

$$
\mathrm{ab}
$$

cd

denote the set of matrices

$$
\left(\begin{array}{lll|ll}
* & \ldots & * & a & b \\
0 & \ldots & 0 & c & d
\end{array}\right)
$$

For the affine polar graph $\Gamma_{e}=V O^{+}(2 e, 2)$, consider the subgraph $\Delta$ induced by the set of all matrices

$$
\left(\begin{array}{lll|ll}
* & \ldots & * & a & b \\
0 & \ldots & 0 & c & d
\end{array}\right)
$$

where $a, b, c, d$ run over $\mathbb{F}_{2}$. The adjacency matrix of the subgraph $\Delta$ is presented by the block-matrix in Figure 7, where $K$ denotes the adjacency matrix of the complete graph on $2^{e-2}$ vertices; $J$ denotes the all-ones matrix of size $2^{e-2} \times 2^{e-2} ; Z$ denotes the all-zeroes matrix of size $2^{e-2} \times 2^{e-2}$.

Switching edges between the cliques $W_{1}, v+W_{1}$ and then between the cliques $V_{1} \cup V_{2}$, $C$ is equivalent to inverting the highlighted entries in Figure 7 . This gives the submatrix of the adjacency matrix of $\Gamma_{e, 2}$ presented in Figure 8. Note that every switched edge connects vertices from the subgraph $\Delta$. This means that the switching preserves all edges having a vertex outside of $\Delta$.

Let $(n, k, \lambda, \mu)$ be the parameters of the affine polar graph $V^{+}(2 e, 2)$ as a strongly regular graph. We have to check that the obtained graph $\Gamma_{e, 2}$ is a strictly Neumaier graph. Note that the vertices

$$
\left(\begin{array}{lll|ll}
* & \ldots & * & * & 1 \\
0 & \ldots & 0 & 1 & *
\end{array}\right)
$$

induce a $2^{e-1}$-regular $2^{e}$-clique in $\Gamma_{e, 2}$ as well as in $\Gamma_{e}$. Let us check that any pair of vertices in $\Gamma_{e, 2}$ is OK, i.e., any two adjacent vertices have $\lambda$ common neighbours. Also, we investigate which values of $\mu$ occur in $\Gamma_{e, 2}$.

Let us consider any two vertices inside of $\Delta$. The notation in the right column of the matrix in Figure 8 means the following. Two block-rows have the same letter if and only if any row from one block-row and any row from the other block-row correspond to non-adjacent vertices having $\mu+2^{e-1}$ common neighbours; two block-rows have the same number if and only if any row from one block-row and any row from the other blockrow correspond to non-adjacent vertices having $\mu-2^{e-1}$ common neighbours. Otherwise, every two non-adjacent vertices corresponding to rows of this submatrix have $\mu$ common neighbours. Any two adjacent vertices have $\lambda$ common neighbours. This means that all pairs of vertices inside of $\Delta$ are OK. 


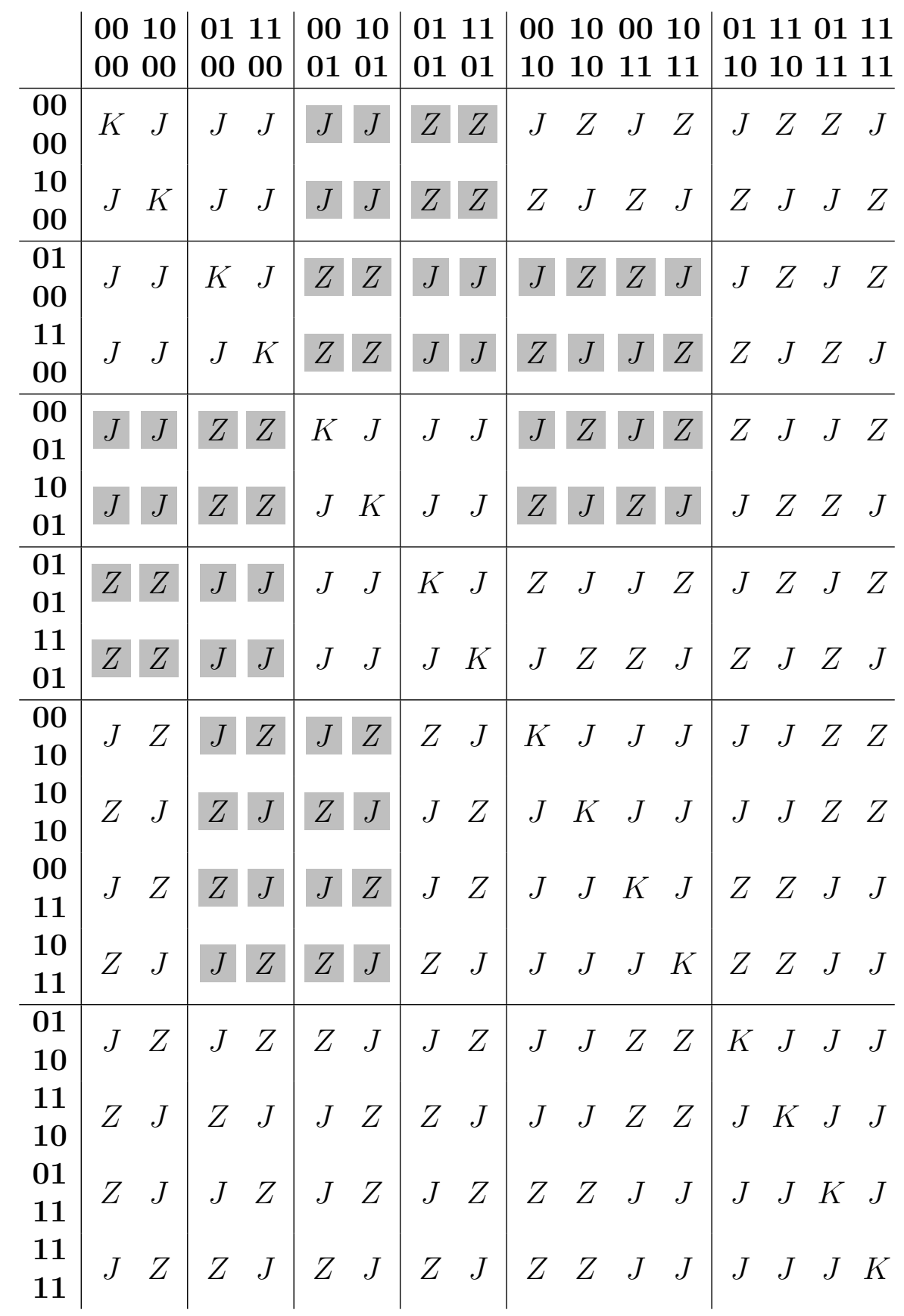

Figure 7. The adjacency matrix, $B_{e}$, of the subgraph $\Delta$ of $\Gamma_{e}$

Let us consider any two vertices outside of $\Delta$. Their neighbours and, consequently, their common neighbours are preserved by the switching. This means that all pairs of vertices outside of $\Delta$ are OK.

Let us consider a vertex $x$ in $\Delta$ and a vertex $y$ outside of $\Delta$. If the neighbours of $x$ are preserved by the switching, then $x, y$ are OK. Assume that the neighbours of $x$ 


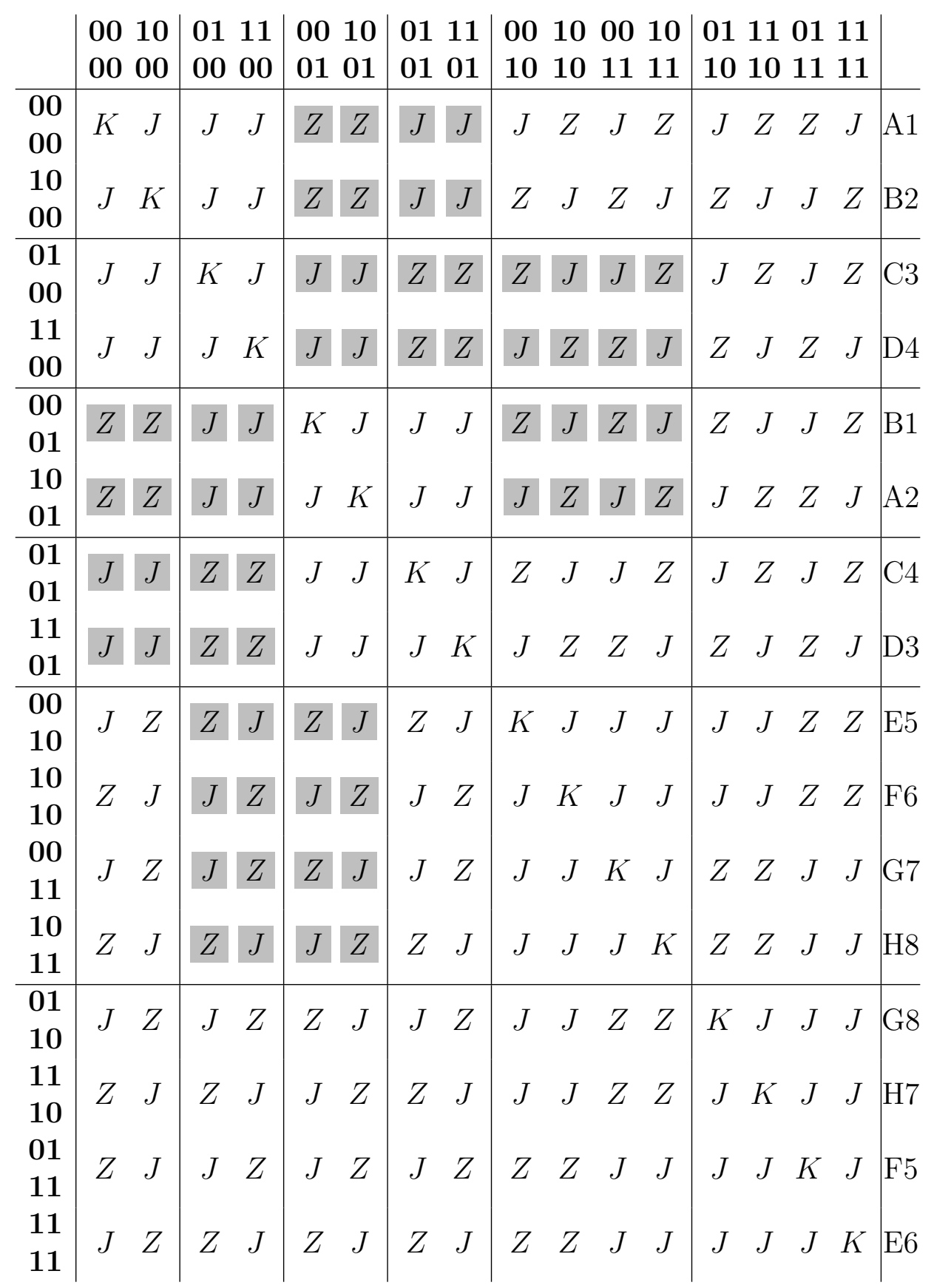

Figure 8. The adjacency matrix, $B_{e, 2}$, of the subgraph $\Delta$ of $\Gamma_{e, 2}$

are switched. Then the vertices $x, y$ are OK since the vertex $y$ is adjacent to half of the vertices of each block of $\Delta$. In fact, the vertex $y$ is given by a matrix

$$
\left(\begin{array}{ccc|cc}
y_{1} & \ldots & y_{2 e-5} & y_{2 e-3} & y_{2 e-1} \\
y_{2} & \ldots & y_{2 e-4} & y_{2 e-2} & y_{2 e}
\end{array}\right)
$$

where there is at least one non-zero among $y_{2}, y_{4}, \ldots, y_{2 e-4}$. Without loss of generality, 
assume that $y_{2}=1$. Let us show that $y$ is adjacent to half of the vertices in a block

$$
\left(\begin{array}{lll|ll}
* & \ldots & * & a & b \\
0 & \ldots & 0 & c & d
\end{array}\right)
$$

We have

$$
\begin{aligned}
y+\left(\begin{array}{ccc|cc}
* & \ldots & * & a & b \\
0 & \ldots & 0 & c & d
\end{array}\right) & =\left(\begin{array}{ccc|cc}
* & \ldots & * & a^{\prime} & b^{\prime} \\
1 & \ldots & y_{2 e-4} & c^{\prime} & d^{\prime}
\end{array}\right) \\
& =\left(\begin{array}{ccc|cc}
0 & \ldots & * & a^{\prime} & b^{\prime} \\
1 & \ldots & y_{2 e-4} & c^{\prime} & d^{\prime}
\end{array}\right) \bigcup\left(\begin{array}{ccc|cc}
1 & \ldots & * & a^{\prime} & b^{\prime} \\
1 & \ldots & y_{2 e-4} & c^{\prime} & d^{\prime}
\end{array}\right) \\
& =Y_{0} \cup Y_{1} .
\end{aligned}
$$

Note that $\left|Y_{0}\right|=\left|Y_{1}\right|$, and the form $Q$ has value 0 on one of the sets $Y_{0}, Y_{1}$ and value 1 on the other. We have proved that the switching preserves the number of common neighbours $x$ and $y$, completing the proof of the theorem.

\section{Concluding remarks}

Let us note that the smallest strictly Neumaier graph can also be constructed as a Cayley graph over $\mathbb{Z}_{2} \times \mathbb{Z}_{8}$ with generating set

$$
\{(0,1),(0,2),(0,4),(0,6),(0,7),(1,1),(1,2),(1,6),(1,7)\} .
$$

There are four known non-isomorphic strictly Neumaier graphs with parameters $(24,8,2 ; 1,4)$, all of which are vertex-transitive. An interesting open problem is to determine all strictly Neumaier graphs with these parameters (up to isomorphism). This will complete the classification of strictly Neumaier graphs on at most 24 vertices.

For $e=3$ and 4, the two generalisations in Section 5 are known to give non-isomorphic graphs. We conjecture that the $i^{t h}$ element of the first sequence of graphs is not isomorphic to the $i^{t h}$ element of the second sequence of graphs, except for the value $i=1$.

Both of the constructions in Section 5 involve taking two pairs of disjoint regular cliques, and carrying out a switching between the cliques in each pair. Starting with the graph $\mathrm{VO}^{+}(6,2)$, it can be shown computationally that any two such consecutive switchings between regular cliques give rise to only two distinct strictly Neumaier graphs, each of which appear in one of the above constructions. We also note that we can continue to apply switchings on disjoint regular cliques, and obtain many new strictly Neumaier graphs with the same parameters. For example, in this way we can show that there are at least 4 non-isomorphic strictly Neumaier graphs with the same parameters as $\mathrm{VO}^{+}(6,2)$. A natural question to ask is how many non-isomorphic strictly Neumaier graphs can we construct in this manner. We hope to use this iterative process to observe prolific constructions of strictly Neumaier graphs, similar to some prolific constructions 
of strongly regular graphs (see Wallis [29], Fon-Der-Flaass [13], Cameron \& Stark [8] and Muzychuk [22]).

The above constructions show that the nexus of a clique in a strictly Neumaier graph is not bounded above by some constant number. However, all known Neumaier graphs contain regular cliques with nexus $2^{j}$, for $j$ a non-negative integer. So we ask if there exist strictly Neumaier graphs containing regular cliques with nexus not a power of two? Finally we ask if we can generalise the above constructions to the case $q$ an arbitrary prime power, which would give strictly Neumaier graphs containing a regular clique with nexus a prime power.

\section{Acknowledgments}

We would like to express our gratitude to Leonard Soicher, Alexander Gavrilyuk and Yaokun $\mathrm{Wu}$ for introducing the authors and their continued support. We are also grateful to Jack Koolen and Gary Greaves for their advice and suggestions on the topics discussed. Finally, we would like to thank Derek Holt and Gordon Royle for providing us with their enumeration of small vertex-transitive edge-regular graphs.

\section{References}

[1] S. Bang, A. Hiraki and J.H. Koolen, Delsarte clique graphs, Europ. J. Combin., 28, 501-516 (2007).

[2] W. Bosma, J. Cannon and C. Playoust, The Magma algebra system. I. The user language, J. Symbolic Comput., 24(3-4), 235-265 (1997).

[3] B. De Bruyn, An Introduction to Incidence Geometry, Frontiers in Mathematics, Birkhäuser Basel (2016).

[4] A. E. Brouwer, A. M. Cohen, and A. Neumaier, Distance-Regular Graphs, SpringerVerlag, Berlin (1989).

[5] A. E. Brouwer and W. H. Haemers, Spectra of Graphs, Springer-Verlag, New York (2012).

[6] A. E. Brouwer and E. E. Shult, Graphs with odd cocliques, Europ. J. Combin., 11, 99-104 (1990).

[7] K. Coolsaet, P. D. Johnson, K. J. Roblee and T. D. Smotzer, Some extremal problems for edge-regular graphs, Ars Combin., 105, 411-418 (2012).

[8] P. J. Cameron and D. Stark, A prolific construction of strongly regular graphs with the n-e.c. property, Electron. J. Combin., 9, \#R31 (2002).

[9] L. C. Chang, The Uniqueness and Non-Uniqueness of the Triangular Association Scheme, In: Science Record, volume 3, Peking Math. Soc., 604-613 (1959).

[10] W. S. Connor, The Uniqueness of the Triangular Association Scheme, Ann. Math. Statist., 29(1), 262-266 (1958). 
[11] P. Delsarte, An algebraic approach to the association schemes of coding theory, Philips Res. Reports Suppl., 10, 143-161 (1973).

[12] M. Erickson, S. Fernando, W.H. Haemers, D. Hardy and J. Hemmeter, Deza graphs: A generalization of strongly regular graphs J. Comb. Des., 7, no. 6, 359-405 (1999).

[13] D. G. Fon-Der-Flaass, New prolific constructions of strongly regular graphs, Adv. Geom. 2(3), 301-306 (2002).

[14] The GAP Group, GAP - Groups, Algorithms, and Programming, 2017, Version 4.8.8.

[15] C. Godsil, Algebraic Combinatorics, Chapman \& Hall, New York (1993).

[16] C. Godsil and G. Royle, Algebraic Graph Theory, Springer-Verlag, New York (2001).

[17] S. V. Goryainov and L. V. Shalaginov, Cayley-Deza graphs with fewer than 60 vertices, Sibirskie Ėlektronnye Matematicheskie Izvestiya, 11, 268-310 (2014).

[18] G. R. W. Greaves and J. H. Koolen, Another construction of edge-regular graphs with regular cliques, Discrete Math., (2018), https://doi.org/10.1016/j.disc.2018. 09.032 .

[19] G. R. W. Greaves and J. H. Koolen, Edge-regular graphs with regular cliques, Europ. J. Combin., 71, 194-201 (2018).

[20] A. J. Hoffman, On the Uniqueness of the Triangular Association Scheme, Ann. Math. Statist., 31(2), 492-497 (1960).

[21] D. Holt and G. Royle, A Census of Small Transitive Groups and Vertex-Transitive Graphs, arXiv:1811.09015.

[22] M. Muzychuk, A generalization of Wallis-Fon-Der-Flaass construction of strongly regular graphs, J. Alg. Combin., 25, 169-187 (2007).

[23] A. Neumaier, Regular Cliques in graphs and Special 1 $\frac{1}{2}$-designs, Finite Geometries and Designs, London Mathematical Society Lecture Note Series, 245-259 (1981).

[24] A. Neumaier, Completely regular codes, Discrete Math., 106/107, 353-360 (1992).

[25] S. S. Shrikhande, On a Characterization of the Triangular Association Scheme, Ann. Math. Statist., 30(1), 39-47 (1959).

[26] S. S. Shrikhande, The Uniqueness of the L2 Association Scheme, Ann. Math. Statist., 30(3), 781-798 (1959).

[27] L. H. Soicher, More on block intersection polynomials and new applications to graphs and block designs, J. Combin. Theory Ser. A, 117(7), 799-809 (2010).

[28] L. H. Soicher, On cliques in edge-regular graphs, J. Algebra, 421, 260-267 (2015).

[29] W. D. Wallis, Construction of strongly regular graphs using affine designs, Bull. Austr. Math. Soc., 4, 41-49 (1971). 